\title{
Flocking with General Local Interaction and Large Population*
}

\author{
CHEN Ge · LIU Zhixin
}

\begin{abstract}
This paper studies a flocking model in which the interaction between agents is described by a general local nonlinear function depending on the distance between agents. The existing analysis provided sufficient conditions for flocking under an assumption imposed on the system's closed-loop states; however this assumption is hard to verify. To avoid this kind of assumption the authors introduce some new methods including large deviations theory and estimation of spectral radius of random geometric graphs. For uniformly and independently distributed initial states, the authors establish sufficient conditions and necessary conditions for flocking with large population. The results reveal that under some conditions, the critical interaction radius for flocking is almost the same as the critical radius for connectivity of the initial neighbor graph.
\end{abstract}

Keywords Consensus, Cucker-Smale model, flocking model, multi-agent systems, random geometric graph.

\section{Introduction}

The flocking, which means the collective coherent motion of a large number of self-propelled organisms, is an amazing phenomenon in nature. It attracts the researchers from diverse fields, including biology, physics, computer science, mathematics and control theory, see [1-6] among many others. In order to investigate the flocking phenomena exhibited in biological systems, the well-known Vicsek model was proposed in [3], in which the heading is updated according to the headings of the corresponding neighbors. Simulation results reveal that the headings of all agents will be almost the same (called consensus or flocking) for large population size. The theoretical study for the flocking behavior of the Vicsek model can be found in [7-10]. Inspired by the Vicsek model, Cucker and Smale also developed a flocking model in which each agent interacts with all other agents and the interaction weights decay according to the agents' distance, and proposed some sufficient conditions for flocking which depend on system parameters only ${ }^{[11]}$. Following their work, the theoretic results with different scenarios, for example, the noisy environment, and the hierarchical structure, are given for the Cucker-Smale model and its variants (see [12-20]).

CHEN Ge $\cdot$ LIU Zhixin

Key Laboratory of Systems and Control \& National Center for Mathematics and Interdisciplinary Sciences, Academy of Mathematics and Systems Science, Chinese Academy of Sciences, Beijing 100190, China.

Email: chenge@amss.ac.cn; Lzx@amss.ac.cn.

* The research was supported by the National Natural Science Foundation of China under Grant No. 11688101, 91634203, 91427304, and 61673373, and the National Key Basic Research Program of China (973 Program) under Grant No. 2016YFB0800404. Part of this paper was presented in the 10th World Congress on Intelligent Control and Automation, pp. 3515-3519, July 6-8, 2012, Beijing, China. 
The assumption of global interaction between agents in the Cucker-Smale model may not be applicable for some practical systems especially for systems with large population size. For example, in [4], Buhl, et al. observed that the individual adjusts its direction to align with its neighbors within an interaction range; In [21], Rosenthal, et al. found the golden shiner (a kind of fish) uses simple, robust measures to assess behavioral changes of the others in neighboring region, and the interaction weight depends on the metric distance and ranked angular area; In [22], Rieu, et al. showed that the spatial correlation of the velocities of Hydra cells decreases to zero rapidly with regarding the distance between cells, and remains zero when the distance is large. For some systems, it may be even impossible to obtain the explicit expression of interaction between agents (see [23]). To be more practical, Martin, et al. proposed a multi-agent model in which the interaction between agents is represented by a general non-negative and non-increasing function ${ }^{[24]}$. Also, they showed that the system will achieve flocking behavior if the maximal perturbation allowed on the relative positions of agents is not bigger than a constant, and the maximum difference among the initial velocities of all

agents is small enough ${ }^{[24]}$. However, how to guarantee the condition of the relative position perturbation is unsolved.

This paper aims at providing some flocking conditions depending on initial state and system parameters only for a multi-agent model with general local interaction. This problem is challenging because the positions and velocities of all agents are coupled. Also, the local interaction between agents are described by a general state-dependent function. These characteristics make that many traditional tools like Lyapunov method cannot be used. Similar to [25-27], we investigate the flocking behavior under random initial states. The large deviations theory is introduced to estimate the maximum degree of the dynamic weighted graphs, and the extension of the random geometric graph theory is employed to estimate the spectral radius of the initial neighbor graphs. We establish sufficient conditions and necessary conditions for the flocking behavior of our multi-agent model. In particular, we reveal that under some conditions, the smallest possible interaction radius for flocking is almost the same as the critical connectivity radius of the initial neighbor graphs.

The rest of this paper is organized as follows. In Section 2, we provide the problem formluation. The main results are given in Section 3. In Section 4, we present the approach to estimating and calculating a key value which characterizes the property of nonlinear functions. The proofs of our main results are put in Section 5. Concluding remarks are made in Section 6 .

\section{Problem Statement}

\subsection{A Nonlinear Flocking Model}

This paper considers a nonlinear discrete-time multi-agent system composed of $n$ spatially distributed agents, each moving in a $d$-dimensional $(d \geq 2)$ Euclidean space $\mathbb{R}^{d}$. Let $\mathcal{V} \triangleq$ $\{1,2, \cdots, n\}$ to be the set of all agents. Set $X_{i}(t) \in \mathbb{R}^{d}$ and $V_{i}(t) \in \mathbb{R}^{d}$ to be the position and velocity of agent $i$ at time $t$ respectively. Following [24] but with some modifications, for any 
time $t \geq 0$ and agent $i \in \mathcal{V}, X_{i}(t)$ and $V_{i}(t)$ are updated according to the following equation,

$$
\left\{\begin{array}{l}
X_{i}(t+1)=X_{i}(t)+V_{i}(t), \\
V_{i}(t+1)=V_{i}(t)+\sum_{j=1}^{n} f_{n}\left(\left\|x_{i}(t)-x_{j}(t)\right\|\right)\left(V_{j}(t)-V_{i}(t)\right),
\end{array}\right.
$$

where the nonlinear function $f_{n}(\cdot)$ denotes the local interaction weight, and $\|\cdot\|$ represents the Euclidean norm. From an intuitive point of view, the larger the distance between agents, the weaker the interaction weight $f_{n}(\cdot)$ should be. Without loss of generality, we assume that $f_{n}(\cdot)$ is a non-increasing integrable function satisfying

$$
f_{n}(x) \begin{cases}>0, & 0 \leq x<r_{n} \\ =0, & x \geq r_{n}\end{cases}
$$

where $r_{n}$ is called the interaction radius. In this paper, the commonly used circular or spherical neighborhood is adopted. The pair of two agents is called neighbors if and only if their distance is less than a pre-defined radius $r_{n}$.

Let $x_{i j}(t)$ and $v_{i j}(t)$ denote the $j$ th element of $X_{i}(t)$ and $V_{i}(t)$, respectively. Denote $X(t):=$ $\left(x_{i j}(t)\right)_{n \times d}$ and $V(t):=\left(v_{i j}(t)\right)_{n \times d}$. The system (1) can be rewritten into the following matrix form:

$$
\left\{\begin{array}{l}
X(t+1)=X(t)+V(t) \\
V(t+1)=P(t) V(t)
\end{array}\right.
$$

where $P(t)=\left(p_{i j}(t)\right)_{n \times n}$ is the weighted average matrix defined by

$$
p_{i j}(t)= \begin{cases}f_{n}\left(\left\|x_{i}(t)-x_{j}(t)\right\|\right), & \text { if } j \neq i, \\ 1-\sum_{j=1, j \neq i}^{n} f_{n}\left(\left\|x_{i}(t)-x_{j}(t)\right\|\right), & \text { otherwise. }\end{cases}
$$

For any $i \in \mathcal{V}$ and $t(=0,1, \cdots)$, we have $\sum_{j=1}^{n} p_{i j}(t)=1$.

The objective of this paper is to investigate the flocking behavior of the system (3) with large population. Follow [24] we say that the system (3) achieves a flocking behavior if the velocities of all agents reach agreement, that is,

$$
\lim _{t \rightarrow \infty} \max _{1 \leq i, j \leq n}\left\|V_{i}(t)-V_{j}(t)\right\|=0 .
$$

\subsection{Random Geometric Graphs}

Following our previous work [27], we assume that the $n$ agents are independently and uniformly distributed in the unit cube $[0,1]^{d}$. Denote $\mathcal{X}_{n}=\left\{X_{1}(0), X_{2}(0), \cdots, X_{n}(0)\right\}$ as the set of initial positions of the $n$ agents. We introduce a random geometric graph $G\left(\mathcal{X}_{n} ; r_{n}\right)$ to describe the neighbor relations between agents at the initial time, with vertex set $\mathcal{V}$ and with undirected edges connecting the pairs $\left\{X_{i}(0), X_{j}(0)\right\}$ satisfying $\left\|X_{i}(0)-X_{j}(0)\right\| \leq r_{n}$; See [28] 
for more properties of random geometric graphs. It is worth pointing out that the positions are not independent and the properties of random geometric graphs cannot be used any more when the agents move around.

In order to state our results clearly, we assume that the interaction radius has the following expression,

$$
\lim _{n \rightarrow \infty}\left(n r_{n}^{d} / \log n\right)=\alpha \in(0, \infty]
$$

Set $R_{c}=R_{c}(n):=\sqrt[d]{\frac{2^{d-1} \log n}{d \pi_{d} n}}$, where $\pi_{d}$ denotes the volume of the unit ball in $\mathbb{R}^{d}$. It is proved that for $d=2, R_{c}$ is the critical connectivity radius of $G\left(\mathcal{X}_{n} ; r_{n}\right)$ in a probability sense (see [29]). The following lemma shows that a similar result holds for $d \geq 3$.

Lemma 2.1 The random geometric graph $G\left(\mathcal{X}_{n} ; r_{n}\right)$ is connected with high probability if $\alpha>\frac{2^{d-1}}{d \pi_{d}}$, and is not connected with high probability if $\alpha<\frac{2^{d-1}}{d \pi_{d}}$, where $\alpha$ is defined by (5).

The proof of Lemma 2.1 is presented in Appendix 6 .

We call $r_{n}$ the super-critical connectivity radius of $G\left(\mathcal{X}_{n} ; r_{n}\right)$ if $\alpha>2^{d-1} /\left(d \pi_{d}\right)$, and we call $r_{n}$ the sub-critical connectivity radius of $G\left(\mathcal{X}_{n} ; r_{n}\right)$ if $\alpha<2^{d-1} /\left(d \pi_{d}\right)$.

\subsection{Large Deviations Techniques}

The large deviations techniques are applied to deal with the influence of the nonlinear interaction weights $f_{n}(\cdot)$. We first introduce some notations.

For a given constant $\delta \geq 0$, define

$$
f_{n, \delta}(x):= \begin{cases}f_{n}(0), & \text { if } x \leq \delta r_{n} \\ f_{n}\left(x-\delta r_{n}\right), & \text { else. }\end{cases}
$$

By the definition of $f_{n, \delta}$, we have $f_{n}=f_{n, 0}$. Let $x_{0}=\left(\frac{1}{2}, \frac{1}{2}, \cdots, \frac{1}{2}\right)$ be the center point of $[0,1]^{d}$, and set

$$
\xi_{n, \delta}:=f_{n, \delta}\left(\left\|X-x_{0}\right\|\right)
$$

where $X$ is a random variable uniformly distributed in $[0,1]^{d}$. For $x \in \mathbb{R}$, define

$$
I_{n, \delta}(x):=\sup _{\theta>0}\left\{\theta x-(n-1) \log \left(E\left[\mathrm{e}^{\theta \xi_{n, \delta}}\right]\right)\right\} .
$$

The function (6) is called a rate function in large deviations theory, see Chapter 1.2 of [30]. By Lemma 2.2.5 in [30], we have

$$
I_{n, \delta}\left\{(n-1) E\left[\xi_{n, \delta}\right]\right\}=0 .
$$

Let $\bar{k}_{n, \delta}$ be $(n-1) f_{n}(0)$ for the case of $\operatorname{Var}\left(\xi_{n, \delta}\right)=E \xi_{n, \delta}^{2}-\left(E \xi_{n, \delta}\right)^{2}=0$ (i.e., $\xi_{n, \delta}=f_{n}(0)$ is a degenerate random variable), and be a solution of the equation $I_{n, \delta}(x)=\log n$ in $((n-$ 1) $\left.E\left[\xi_{n, \delta}\right], \infty\right)$ for the case of $\operatorname{Var}\left(\xi_{n, \delta}\right)>0$. We will show that the solution uniquely exists in Section 4. For the simplicity of expression, we denote $\xi_{n}=\xi_{n, 0}, I_{n}=I_{n, 0}$, and $\bar{k}_{n}=\bar{k}_{n, 0}$. 


\subsection{Notation}

In this paper, we investigate the flocking behavior of the system (3) on the probability space $(\Omega, \mathcal{F}, P)$, where the sample space $\Omega=[0,1]^{d n}$, and the $\sigma$-algebra $\mathcal{F}$ is the collection of all the Borel subsets of $\Omega$. We say that a sequence of events $A_{n}(n \geq 1)$ occurs with high probability (w.h.p.) if $\lim _{n \rightarrow \infty} P\left(A_{n}\right)=1$.

A square matrix $M=\left(m_{i j}\right)_{n \times n}$ is called stochastic, if all elements $m_{i j}$ is nonnegative and for $1 \leq i \leq n, \sum_{j=1}^{n} m_{i j}=1$. For a matrix $A \in R^{n \times d}$, the Frobenius norm $\|\cdot\|_{F}$ and

the max norm $\|\cdot\|_{\max }$ of the matrix $A$ are, respectively, defined as $\|A\|_{F}=\sqrt{\sum_{i, j} a_{i j}^{2}}$ and $\|A\|_{\max }:=\max _{i, j}\left|a_{i j}\right|$.

For two positive scalar sequences $g_{1}(n)$ and $g_{2}(n)$, we say that (i) $g_{1}(n)=O\left(g_{2}(n)\right)$ if there exists a constant $c>0$ and a value $n_{0}>0$ such that $g_{1}(n) \leq c g_{2}(n)$ for any $n \geq n_{0}$; (ii) $g_{1}(n)=\Theta\left(g_{2}(n)\right)$ if there exist positive constants $c_{1}$ and $c_{2}$ and a value $n_{0}>0$ such that $c_{1} g_{2}(n) \leq g_{1}(n) \leq c_{2} g_{2}(n)$ for any $n \geq n_{0}$; (iii) $g_{1}(n)=o\left(g_{2}(n)\right)$ if $\lim _{n \rightarrow \infty} \frac{g_{1}(n)}{g_{2}(n)}=0$.

\section{Main Results}

In this paper, we proceed with our analysis under the system (3) with the following assumptions on the initial states of all agents and the interaction function $f_{n}(\cdot)$ :

A1) The initial positions $\left\{X_{i}(0)\right\}_{i=1}^{n}$ are independently and uniformly distributed in $[0,1]^{d}$.

A2) The nonlinear interaction weight $f_{n}(\cdot)$ is a non-increasing integrable function satisfying (2), and the interaction radius $r_{n}$ satisfies (5).

To avoid repetitive description we do not state the above assumptions in our results.

\subsection{Sufficient Conditions for Flocking}

Let $V_{0}:=\frac{1}{n} \sum_{i=1}^{n} V_{i}(0)$ and $\bar{V}:=\left(V_{0}^{\mathrm{T}}, V_{0}^{\mathrm{T}}, \cdots, V_{0}^{\mathrm{T}}\right)^{\mathrm{T}} \in \mathbb{R}^{n \times d}$. Denote

$$
\mathcal{L}(V(0))=\|V(0)-\bar{V}\|_{\max }\left[\log \left(\frac{\|V(0)-\bar{V}\|_{F}}{\|V(0)-\bar{V}\|_{\max }}\right)+1\right] .
$$

The sufficient condition for flocking can be stated as follows.

Theorem 3.1 Suppose that the parameter $\alpha$ defined by (5) satisfies $\alpha>\frac{2^{d-1}}{d \pi_{d}}$, and that there exist positive constants $\delta$ and $\varepsilon$ such that $\bar{k}_{n, \delta} \leq 1-\varepsilon$ holds for large $n$. Then flocking is achieved w.h.p. if one of the following two conditions holds:

(i) $\alpha \varepsilon^{d} \leq(d+3)^{d / 2}$, and

$$
\mathcal{L}(V(0)) \leq c \delta n^{2} r_{n} \cdot \min \left\{r_{n}^{2 d+2} f_{n}^{2}\left((\delta+\varepsilon) r_{n}\right), \frac{f_{n}^{2}\left(R_{c}+(\delta+\varepsilon) r_{n}\right)}{(\log n)^{2 d /(d-1)}}\right\} ;
$$

(ii) $\alpha \varepsilon^{d}>(d+3)^{d / 2}$, and

$$
\mathcal{L}(V(0)) \leq c \delta f_{n}^{2}\left[(\delta+\varepsilon) r_{n}\right] n^{2} r_{n} \cdot \min \left\{r_{n}^{2 d+2}, 1\right\},
$$

where $c$ is a positive constant depending on $d, \varepsilon$ and $\alpha^{\dagger}$.

\footnotetext{
${ }^{\dagger}$ Throughout this paper, a constant $c$ depending on $\alpha$ means that $c$ depends on $\alpha$ only for $\alpha<\infty$, and will not depend on $\alpha$ for $\alpha=\infty$.
} 
The proof of Theorem 3.1 is put in Subsection 5.2.

Remark 3.2 The value of $\bar{k}_{n, \delta}$ in Theorem 3.1 can be calculated by solving Equation (16), and some theoretical results for $\bar{k}_{n, \delta}$ can also be obtained, see Proposition 4.1 in Section 4.

Remark 3.3 It is clear that $\mathcal{L}(V(0))$ defined via (7) satisfies $\mathcal{L}(V(0)) \leq\left(\frac{\log n}{2}+1\right)$ $\max _{i, j}\left|v_{i j}(0)\right|$. Hence, Theorem 3.1 still holds if $\mathcal{L}(V(0))$ in (8) and (9) is replaced by $\left(\frac{\log n}{2}+1\right)$ $\max _{i, j}\left|v_{i j}(0)\right|$, which means that under some conditions on the neighborhood radius and the interaction weights, the system (3) can reach flocking if the initial velocities are suitably small.

In fact, under some further conditions on $f_{n}$, the parameter $\delta$ in the condition of Theorem 3.1 can be removed, see the following corollary.

Corollary 3.4 Let $\alpha>\frac{2^{d-1}}{d \pi_{d}}$ and $r_{n}=o(1)$. Suppose that there exists a constant $\varepsilon>0$ such that $\bar{k}_{n} \leq 1-\varepsilon$ for large $n$, and

$$
\inf _{n}\left(\frac{1}{f_{n}(0)} \int_{0}^{1} f_{n}\left(r_{n} y\right) y^{d-1} d y\right)=c_{0}>0 .
$$

Then the system (3) reaches flocking w.h.p., if one of the following two conditions holds:

(i) $\alpha \varepsilon^{d} \leq(d+3)^{d / 2}$ and

$$
\mathcal{L}(V(0)) \leq c n^{2} r_{n} \cdot \min \left\{r_{n}^{2 d+2} f_{n}^{2}\left(c_{1} \varepsilon r_{n}\right), \frac{f_{n}^{2}\left(R_{c}+c_{1} \varepsilon r_{n}\right)}{(\log n)^{2 d /(d-1)}}\right\}
$$

(ii) $\alpha \varepsilon^{d}>(d+3)^{d / 2}$ and $\mathcal{L}(V(0)) \leq c n^{2} r_{n}^{2 d+3} f_{n}^{2}\left(c_{1} \varepsilon r_{n}\right)$, where $c=c\left(d, \varepsilon, c_{0}, \alpha\right)$ and $c_{1}=$ $c_{1}\left(d, c_{0}, \alpha\right)$ are two positive constants.

The proof of Corollary 3.4 is put in Subsection 5.2.

Remark 3.5 From an intuitive point of view, the condition (10) means that the interaction weight $f_{n}(\cdot)$ steadily decreases to zero, and cannot decay very fast.

In the following, we provide an example to illustrate the result of Corollary 3.4.

Example 3.6 Let $\beta>\frac{1}{d}, c^{\prime} \in(0,1]$ and $\gamma>0$ be three constants. Set

$$
f_{n}(x):= \begin{cases}c_{n}\left(1-x^{\gamma} / r_{n}^{\gamma}\right), & \text { if } x \leq r_{n} \\ 0, & \text { otherwise }\end{cases}
$$

where $r_{n}=n^{-1 / d} \log ^{\beta} n, c_{n}=\frac{c^{\prime}}{\pi_{d} \log ^{d \beta} n}$. It is easy to verify that $f_{n}$ satisfies (10). Recalling that $\xi_{n}=\xi_{n, 0}=f_{n}\left(\left\|X-x_{0}\right\|\right)$, we obtain the following inequality:

$$
E\left[\xi_{n}\right]=\int_{0}^{r_{n}} c_{n}\left(1-r_{n}^{-\gamma} x^{\gamma}\right) d \pi_{d} x^{d-1} d x=\frac{\gamma c_{n} \pi_{d} r_{n}^{d}}{\gamma+d} \leq \frac{\gamma}{(\gamma+d) n} .
$$

Using the following Proposition 4.1 (iii), we have for large $n, \bar{k}_{n}=n E\left[\xi_{n}\right](1+o(1))<1-\frac{d}{2(\gamma+d)}$. Thus, by Remark 3.3 and Corollary 3.4 (ii), the system (3) reaches flocking w.h.p., if the velocity satisfies $\max _{i, j}\left|v_{i j}(0)\right| \leq c n^{\frac{-3}{d}}(\log n)^{3 \beta-1}$ with $c>0$ being a positive constant. 
We also give some simulations for Theorem 3.1 and Corollary 3.4. Assume the space dimension $d=2$, the interaction radius $r_{n}=\sqrt{\alpha \log n / n}$ with $\alpha$ being a positive constant, and the weight function

$$
f_{n}(x)= \begin{cases}\frac{1-x / r_{n}}{\alpha \pi \log n}, & \text { if } x \leq r_{n} \\ 0, & \text { otherwise. }\end{cases}
$$

If $\alpha>1 / \pi$, we can get

$$
n^{2} r_{n} \cdot \min \left\{r_{n}^{6} f_{n}^{2}\left(\varepsilon r_{n}\right), \frac{f_{n}^{2}\left(\sqrt{\log n /(\pi n)}+\varepsilon r_{n}\right)}{\log ^{4} n}\right\}=n^{2} r_{n}^{7} f_{n}^{2}\left(\varepsilon r_{n}\right)=\Theta\left(\frac{\log n}{n}\right)^{3 / 2},
$$

where $\varepsilon$ is a small positive constant. The initial positions $\left\{X_{i}(0)\right\}_{i=1}^{n}$ are independently and uniformly chosen from the area $[0,1]^{2}$, and the initial velocities are set to be

$$
V_{i}(0)=\left\{\begin{array}{ll}
\left(-v^{\prime} n^{-\frac{3}{2}} \log ^{\frac{1}{2}} n, 0\right), & \text { if } X_{i 1}(0) \leq 1 / 2 \\
\left(v^{\prime} n^{-\frac{3}{2}} \log ^{\frac{1}{2}} n, 0\right), & \text { otherwise }
\end{array}, \quad \forall 1 \leq i \leq n,\right.
$$

where $v^{\prime}$ is a positive constant. By (7) we can obtain

$$
\mathcal{L}(V(0)) \approx v^{\prime} n^{-\frac{3}{2}} \log ^{\frac{1}{2}} n(\log n+1)=\Theta\left(n^{-\frac{3}{2}} \log ^{\frac{3}{2}} n\right) .
$$

Simulations are carried out by choosing $n=600$, and the results are shown in Figure 1. It is shown that there is a demarcation line $v_{c}^{\prime}(\alpha)$ concerning with $v^{\prime}$ and $\alpha$ between the behaviors of flocking and no flocking. From (11), (12) and Figure 1, the sufficient conditions for flocking in Theorem 3.1 and Corollary 3.4 are tight in the order under this kind of interaction functions.

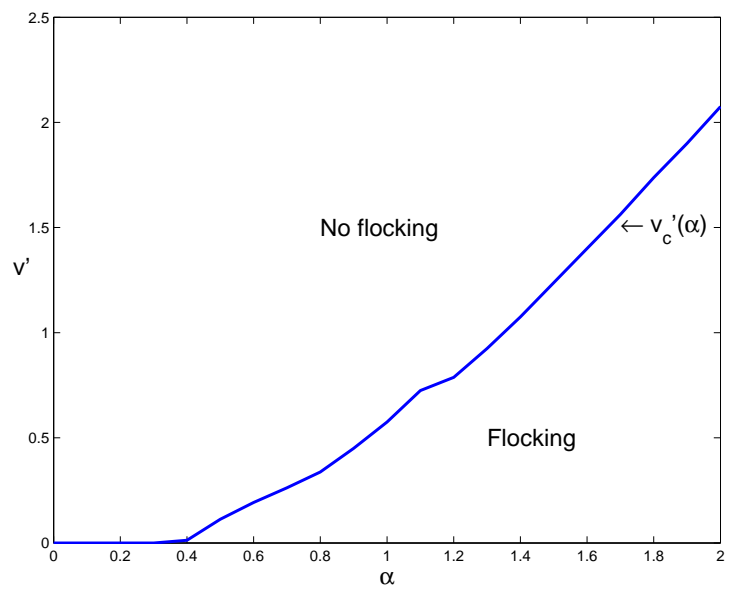

Figure 1 There is a demarcation line $v_{c}^{\prime}(\alpha)$ concerning with $v^{\prime}$ and $\alpha$. The flocking behavior can be reached below the line, while cannot be reached above the line. 


\subsection{Necessary Conditions for Flocking}

By Remark 3.3, we see that for small initial velocity, the connectivity of neighbor graphs can be maintained, and consequently the flocking behavior can be achieved. However, when the initial velocities become larger and larger, the connectivity of the dynamical neighbor graphs may be lost, even though the initial graph is connected. For such a case, the investigation for flocking becomes much harder. In order to simplify the analysis for the dependency of the flocking conditions on initial velocities of the agents, we introduce the definition $v$-flocking as follows.

Definition 3.7 Let $v>0$ be a constant. If the system (3) reaches flocking for any initial velocities satisfying $\max _{i \in \mathcal{V}}\left\|V_{i}(0)\right\| \leq v$, then we say the system reaches $v$-flocking.

From Definition 3.7, we see that the $v$-flocking behavior monotonically depends on $v$. For any initial positions of the agents, there exists a critical value $v_{c}$ such that the $v$-flocking can be achieved for $v<v_{c}$, and cannot be achieved for $v>v_{c}$. The investigation of $v_{c}$ is an interesting but very hard topic, and it falls into our future research.

The necessary conditions for $v$-flocking are presented as follows.

Theorem 3.8 (i) If $\alpha<\frac{2^{d-1}}{d \pi_{d}}$, then the system (3) cannot achieve v-flocking w.h.p. for any $v>0$.

(ii) If $\alpha \geq \frac{2^{d-1}}{d \pi_{d}}$ and $r_{n}=o(1)$, then the system (3) cannot reach $\left(2^{-d-1} \bar{k}_{n} r_{n}\right)$-flocking w.h.p. for $\bar{k}_{n}=\Theta(1)$ and $\bar{k}_{n}<2^{d}$, and also cannot reach $\left(\frac{1}{2} \bar{k}_{n} r_{n}\right)$-flocking w.h.p. for $\bar{k}_{n}=o(1)$.

The proof of Theorem 3.8 is put in Subsection 5.3.

Under the definition of $v$-flocking, the sufficient conditions for flocking can be stated in a more clear manner.

Corollary 3.9 Assume that $\alpha>\frac{2^{d-1}}{d \pi_{d}}$ and $r_{n}=o(1)$, and that there exists a constant $\varepsilon>0$ such that for large $n$, (i) $\varepsilon \leq \bar{k}_{n} \leq 1-\varepsilon$; (ii) $f_{n}\left(\varepsilon r_{n}\right)>\varepsilon f_{n}(0)$; and (iii) $f_{n}\left(R_{c}+\varepsilon r_{n}\right)>$ $r_{n}^{2}(\log n)^{2 d /(d-1)} n^{-2}$. Then the system (3) reaches $\left(c r_{n}^{3}(\log n)^{-1}\right)$-flocking w.h.p., where $c=$ $c(d, \varepsilon, \alpha)>0$ is a constant.

The above corollary can be directly deduced from Corollary 3.4.

From Theorem 3.8 (i) and Corollary 3.9, we see that in a probability sense, $R_{c}$ can be considered as the smallest possible interaction radius for flocking, and also the critical interaction radius for $v$-flocking with $v \leq c r_{n}^{3}(\log n)^{-1}$. We illustrate the result of Theorem 3.8 and Corollary 3.9 in Figure 2 where the interaction function $f_{n}(\cdot)$ satisfies the conditions of Corollary 3.9.

\section{Calculation and Estimation of $\bar{k}_{n, \delta}$}

In this section, we present the approach to the estimation and calculation of $\bar{k}_{n, \delta}$. We only consider the case of $\operatorname{Var}\left(\xi_{n, \delta}\right)>0$. Note that $I_{n, \delta}\left((n-1) E\left[\xi_{n, \delta}\right]\right)=0$, and

$$
\lim _{x \rightarrow \infty} I_{n, \delta}(x) \geq \lim _{x \rightarrow \infty}\left\{x-(n-1) \log \left(E\left[\mathrm{e}^{\xi_{n, \delta}}\right]\right)\right\}=\infty .
$$


By the continuity of $I_{n, \delta}(x)$, we see that the solution of the equation $I_{n, \delta}(x)=\log n$ in $((n-$ 1) $\left.E\left[\xi_{n, \delta}\right], \infty\right)$ exists. Furthermore, by Cauchy-Schwarz inequality, we have

$$
\frac{d^{2}}{d^{2} \theta} \log \left(E\left[\mathrm{e}^{\theta \xi_{n, \delta}}\right]\right)=\frac{E\left[\mathrm{e}^{\theta \xi_{n, \delta}}\right] E\left[\xi_{n, \delta}^{2} \mathrm{e}^{\theta \xi_{n, \delta}}\right]-\left(E\left[\xi_{n, \delta} \mathrm{e}^{\theta \xi_{n, \delta}}\right]\right)^{2}}{\left(E\left[\mathrm{e}^{\theta \xi_{n, \delta}}\right]\right)^{2}}>0 .
$$

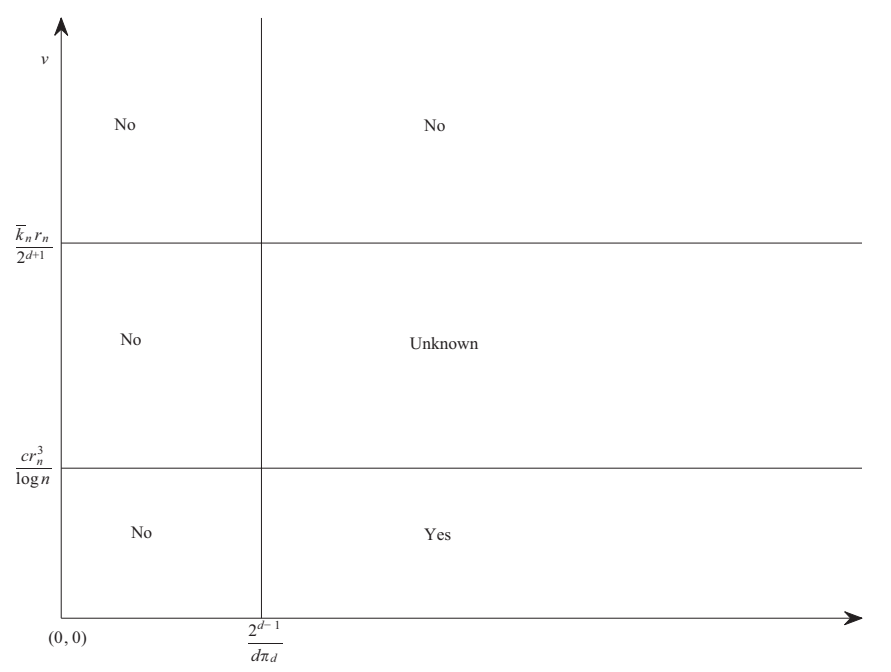

Figure 2 "Yes" (or "No") means that in a probability sense, the system (3) can (cannot) reach $v$-flocking, and "unknown" means that we cannot judge whether the system reaches $v$-flocking

Hence, the following equation

$$
\frac{d}{d \theta}\left\{\theta x-(n-1) \log \left(E\left[\mathrm{e}^{\theta \xi_{n, \delta}}\right]\right)\right\}=x-\frac{(n-1) E\left[\xi_{n, \delta} \mathrm{e}^{\theta \xi_{n, \delta}}\right]}{E\left[\mathrm{e}^{\theta \xi_{n, \delta}}\right]}=0
$$

has a unique solution $\theta^{*}(x)$ for any $x>0$. Moreover, by (13), the right-hand side of (15) is strictly monotonically increasing. Thus, the equation

$$
\begin{aligned}
\log n=I_{n, \delta}(x) & =\theta^{*}(x) x-(n-1) \log \left(E\left[\mathrm{e}^{\theta^{*}(x) \xi_{n, \delta}}\right]\right) \\
& =\theta^{*}(x) \frac{(n-1) E\left[\xi_{n, \delta} \mathrm{e}^{\theta^{*}(x) \xi_{n, \delta}}\right]}{E\left[\mathrm{e}^{\theta^{*}(x) \xi_{n, \delta}}\right]}-(n-1) \log \left(E\left[\mathrm{e}^{\theta^{*}(x) \xi_{n, \delta}}\right]\right)
\end{aligned}
$$

with respect to $\theta^{*}(x)$ also has a unique solution $\bar{\theta}_{n, \delta}$. By (14), we know that $\bar{k}_{n, \delta}$ is the unique solution of $I_{n, \delta}(x)=\log n$ in $\left((n-1) E\left[\xi_{n, \delta}\right], \infty\right)$. Combining (15) with (14), we see that $\bar{k}_{n, \delta}$ and $\bar{\theta}_{n, \delta}$ can be obtained by solving the following equations,

$$
\frac{(n-1) E\left[\xi_{n, \delta} \mathrm{e}^{\bar{\theta}_{n, \delta} \xi_{n, \delta}}\right]}{E\left[\mathrm{e}^{\bar{\theta}_{n, \delta} \xi_{n, \delta}}\right]}=\frac{\log n+(n-1) \log \left(E\left[\mathrm{e}^{\bar{\theta}_{n, \delta} \xi_{n, \delta}}\right]\right)}{\bar{\theta}_{n, \delta}}=\bar{k}_{n, \delta} .
$$


Furthermore, if $f_{n}$ does not decay very fast, there are some theoretical results to estimate $\bar{k}_{n, \delta}, \bar{k}_{n}\left(=: \bar{k}_{n, 0}\right)$ and $\bar{\theta}_{n}\left(=: \bar{\theta}_{n, 0}\right)$.

Proposition 4.1 Suppose that $r_{n}=o(1)$, and $f_{n}$ satisfies (10). Let $c_{0}$ be the constant appearing in (10). Then the following results hold:

(i) $\bar{k}_{n}=\Theta\left(n r_{n}^{d} f_{n}(0)\right)$ and $\bar{\theta}_{n}=O\left(1 / f_{n}(0)\right)$;

(ii) There exists a constant $c_{1}=c_{1}\left(d, \alpha, c_{0}\right)>0$, such that for any $\delta \in(0,1), \bar{k}_{n, \delta} \leq$ $\left(1+c_{1} \delta\right) \bar{k}_{n}$

(iii) For the case of $\alpha=\infty, \bar{k}_{n}=n E\left[\xi_{n}\right](1+o(1))$ and $\bar{\theta}_{n}=o\left(1 / f_{n}(0)\right)$.

The proof of Proposition 4.1 is in Appendix 6.

Using a similar method as that of Proposition 4.1, we obtain the following results.

Corollary 4.2 Suppose $r_{n}=o(1)$. Then for any constant $\delta>0$, we have $\bar{k}_{n, \delta}=$ $\Theta\left(n r_{n}^{d} f_{n}(0)\right)$ and $\bar{\theta}_{n, \delta}=O\left(1 / f_{n}(0)\right)$. If $\alpha=\infty$, then $\bar{k}_{n, \delta}=n E\left[\xi_{n, \delta}\right](1+o(1))$ and $\bar{\theta}_{n, \delta}=$ $o\left(1 / f_{n}(0)\right)$.

In the following, we present two examples to show how to calculate the value of $\bar{k}_{n}$. For this, we introduce some notations in large deviations theory. Define $H:[0, \infty) \rightarrow \mathbb{R}$ by $H(0)=1$ and

$$
H(a)=1-a+a \log a, \quad a>0 .
$$

Note that $H(1)=0$ and the unique turning point of $H$ is the minimum at 1 . Also $H(a) / a$ is increasing on $(1, \infty)$. Let $H_{-}^{-1}:[0,1] \rightarrow[0,1]$ be the unique inverse of the restriction of $H$ to $[0,1]$, and let $H_{+}^{-1}:[0, \infty) \rightarrow[1, \infty)$ be the inverse of the restriction of $H$ to $[1, \infty)$.

Example 4.3 Set $r_{n}=c n^{-1 / d}(\log n)^{1 / d}$ and

$$
f_{n}(x):= \begin{cases}b_{n}, & x \leq r_{n}, \\ 0, & x>r_{n},\end{cases}
$$

where $c$ and $b_{n}$ are positive constants. Recall that $\xi_{n}=\xi_{n, 0}=f_{n}\left(\left\|X-x_{0}\right\|\right)$, where $x_{0}=$ $\left(\frac{1}{2}, \frac{1}{2}, \cdots, \frac{1}{2}\right) \in \mathbb{R}^{d}$ and $X$ is a random variable distributed uniformly in $[0,1]^{d}$. To solve (16) we first calculate the following equation,

$$
\begin{aligned}
E\left[\mathrm{e}^{\bar{\theta}_{n} \xi_{n}}\right] & =P\left(\left\|X-x_{0}\right\| \leq r_{n}\right) \mathrm{e}^{\bar{\theta}_{n} b_{n}}+P\left(\left\|X-x_{0}\right\|>r_{n}\right) \\
& =1+\left(\mathrm{e}^{\bar{\theta}_{n} b_{n}}-1\right) \pi_{d} c^{d} n^{-1} \log n .
\end{aligned}
$$

Similarly,

$$
E\left[\xi_{n} \mathrm{e}^{\bar{\theta}_{n} \xi_{n}}\right]=\mathrm{e}^{\bar{\theta}_{n} b_{n}} \pi_{d} c^{d} b_{n} n^{-1} \log n .
$$

By Proposition 4.1 (i), we have $\bar{\theta}_{n} b_{n}=\bar{\theta}_{n} f_{n}(0)=O(1)$. Then by (17), we obtain $E\left[\mathrm{e}^{\bar{\theta}_{n} \xi_{n}}\right]=$ $1+o\left(n^{-1 / 2}\right)$ and

$$
\log E\left[\mathrm{e}^{\bar{\theta}_{n} \xi_{n}}\right]=\left(\mathrm{e}^{\bar{\theta}_{n} b_{n}}-1\right) \pi_{d} c^{d} n^{-1} \log n+O\left(n^{-2} \log ^{2} n\right) .
$$


Substituting (18) and (19) into (16), we have

$$
\bar{\theta}_{n} b_{n} \mathrm{e}^{\bar{\theta}_{n} b_{n}}-\mathrm{e}^{\bar{\theta}_{n} b_{n}}=\left(\frac{1}{\pi_{d} c^{d}}-1\right)\left(1+o\left(n^{-1 / 2}\right)\right)+o\left(n^{-1 / 2}\right) .
$$

By the definition of $H_{+}^{-1}$, we see that $\log \left(H_{+}^{-1}\left(\frac{1}{\pi_{d} c^{d}}\right)\right)$ is the unique solution of the equation $x \mathrm{e}^{x}-$ $\mathrm{e}^{x}=\frac{1}{\pi_{d} c^{d}}-1$ with respect to $x$. Substituting this into $(20)$ we have $\bar{\theta}_{n}=\frac{1}{b_{n}} \log \left(H_{+}^{-1}\left(\frac{1}{\pi_{d} c^{d}}\right)\right)(1+$ $o(1))$. Furthermore, by (16) we have $\bar{k}_{n}=H_{+}^{-1}\left(\frac{1}{\pi_{d} c^{d}}\right) \pi_{d} c^{d} b_{n} \log n(1+o(1))$.

Example 4.4 Let $r_{n}=c n^{-1 / d}(\log n)^{1 / d}$ and

$$
f_{n}(x):= \begin{cases}b_{n}\left(1-r_{n}^{-1} x\right), & x \leq r_{n} \\ 0, & x>r_{n}\end{cases}
$$

where $c$ and $b_{n}$ are positive constants. Similar to (17) and (18) we have

$$
\begin{aligned}
E\left[\mathrm{e}^{\bar{\theta}_{n} \xi_{n}}\right]= & 1-\pi_{d} r_{n}^{d}+\int_{0}^{r_{n}} \mathrm{e}^{\bar{\theta}_{n} b_{n}\left(1-r_{n}^{-1} x\right)} d \pi_{d} x^{d-1} d x \\
= & 1-\pi_{d} r_{n}^{d}+d \pi_{d} r_{n}^{d} \int_{0}^{1} \mathrm{e}^{\bar{\theta}_{n} b_{n} y}(1-y)^{d-1} d y \\
E\left[\xi_{n} \mathrm{e}^{\bar{\theta}_{n} \xi_{n}}\right] & =\int_{0}^{r_{n}} b_{n}\left(1-r_{n}^{-1} x\right) \mathrm{e}^{\bar{\theta}_{n} b_{n}\left(1-r_{n}^{-1} x\right)} d \pi_{d} x^{d-1} d x \\
& =d \pi_{d} r_{n}^{d} b_{n} \int_{0}^{1} y \mathrm{e}^{\bar{\theta}_{n} b_{n} y}(1-y)^{d-1} d y .
\end{aligned}
$$

By Proposition 4.1 (i), we have $\bar{\theta}_{n} b_{n}=\bar{\theta}_{n} f_{n}(0)=O(1)$. Similar to (20) we have

$$
\begin{aligned}
& \bar{\theta}_{n} b_{n} \int_{0}^{1} y \mathrm{e}^{\bar{\theta}_{n} b_{n} y}(1-y)^{d-1} d y-\int_{0}^{1} \mathrm{e}^{\bar{\theta}_{n} b_{n} y}(1-y)^{d-1} d y \\
= & \left(\frac{1}{d \pi_{d} c^{d}}-\frac{1}{d}\right)\left(1+o\left(n^{-1 / 2}\right)\right)+o\left(n^{-1 / 2}\right) .
\end{aligned}
$$

Let $x^{*}$ be the unique solution of the equation

$$
x \int_{0}^{1} y \mathrm{e}^{x y}(1-y)^{d-1} d y-\int_{0}^{1} \mathrm{e}^{x y}(1-y)^{d-1} d y=\frac{1}{d \pi_{d} c^{d}}-\frac{1}{d}
$$

with respect to $x$. Substituting $x^{*}$ into $(21)$ we can get $\bar{\theta}_{n}=\frac{x^{*}}{b_{n}}(1+o(1))$. Substituting this into (16) we obtain

$$
\bar{k}_{n}=d \pi_{d} c^{d} b_{n} \log n\left(\int_{0}^{1} y \mathrm{e}^{x^{*} y}(1-y)^{d-1} d y\right)(1+o(1)) .
$$

\section{Proofs of Main Results}

\subsection{Key Lemmas}

In order to prove the main results of this paper, we first present some key lemmas. By (3) we have $V(t+1)=P(t) \cdots P(0) V(0)$. To study the flocking behavior, we need to deal with 
the convergence of the matrix product $P(t) \cdots P(0)$. Compared with our previous work [27], a crucial point lies in the fact that the weighted average matrix $P(t)$ is determined by the nonlinear function $f_{n}(\cdot)$ and the distance between agents. We apply the large deviations techniques to deal with this.

Introduce the maximum weighted degree of all agents at time $t$ as

$$
\Delta_{n}(t):=\max _{1 \leq i \leq n}\left\{\sum_{1 \leq j \leq n, j \neq i} f_{n}\left(\left\|X_{i}(t)-X_{j}(t)\right\|\right)\right\} .
$$

Note that $f_{n}(\cdot)$ is a non-increasing function. If there exists a positive constant $\delta$ such that

$$
\left\|X_{i}(t)-X_{j}(t)\right\| \geq\left\|X_{i}(0)-X_{j}(0)\right\|-\delta r_{n},
$$

then by the definition of $f_{n, \delta}(\cdot)$ we have

$$
f_{n}\left(\left\|X_{i}(t)-X_{j}(t)\right\|\right) \leq f_{n, \delta}\left(\left\|X_{i}(0)-X_{j}(0)\right\|\right) .
$$

Thus, we have the following lemma of $\Delta_{n}(t)$.

Lemma 5.1 Assume that there exists a positive constant $\delta$, such that

$$
\Delta_{n, \delta}:=\max _{1 \leq i \leq n}\left\{\sum_{1 \leq j \leq n, j \neq i} f_{n, \delta}\left(\left\|X_{i}(0)-X_{j}(0)\right\|\right)\right\} \leq 1,
$$

and that the inequality (22) holds for all $i$ and $j$ and all $t \geq 0$, then we have $\Delta_{n}(t) \leq 1$.

It is clear that under the conditions of Lemma 5.1, $P(t)(t \geq 0)$ is a stochastic matrix. The stochastic matrices may bring convenience for the convergence property of $P(t) \cdots P(0)$.

For $\Delta_{n, \delta}$, we have the following results.

Lemma 5.2 Given a constant $\delta \geq 0$, suppose that $\operatorname{Var}\left(\xi_{n, \delta}\right)>0$ holds. Then,

$$
\Delta_{n, \delta} \leq \bar{k}_{n, \delta}(1+o(1)), \quad \text { w.h.p. }
$$

The large deviations techniques are used in the proof of Lemma 5.2, and the proof details are put in Appendix 6.

In the following, we study the eigenvalues of the matrix $P(t)$. Under the conditions of Lemma 5.1, $P(t)(t \geq 0)$ is a symmetric stochastic matrix. Thus, the eigenvalues of $P(t)$ are all real numbers. We denote $\lambda_{i}(t)=\lambda_{i}(t, n)$ as the $i$-largest eigenvalues of $P(t)$, and arrange all eigenvalues according to the following order,

$$
1=\lambda_{1}(t) \geq \lambda_{2}(t) \geq \cdots \geq \lambda_{n}(t) \geq-1 .
$$

The essential spectral radius $\bar{\lambda}(t)$ of $P(t)$ is defined as $\bar{\lambda}(t)=\bar{\lambda}(t, n):=\max \left\{\left|\lambda_{2}(t)\right|,\left|\lambda_{n}(t)\right|\right\}$, which plays a key role for the convergence of the matrix product $P(t) \cdots P(0)$.

Lemma 5.3 Assume that $\alpha$ defined by (5) satisfies $\alpha>\frac{2^{d-1}}{d \pi_{d}}$, and that there exists a constant $\delta>0$, such that w.h.p. the following inequality holds,

$$
\sup _{i, j, t}\left\{\left\|X_{i}(t)-X_{j}(t)-X_{i}(0)+X_{j}(0)\right\|\right\} \leq \delta r_{n} .
$$


Furthermore, for any given constant $\varepsilon>0, \bar{k}_{n, \delta}$ satisfies $\bar{k}_{n, \delta} \leq 1-\varepsilon$. Then the essential spectral radius $\bar{\lambda}(t)(t>0)$ satisfies the following inequalities:

(i) If $\alpha \varepsilon^{d} \leq(d+3)^{d / 2}$, then w.h.p.

$$
\bar{\lambda}(t) \leq 1-c n^{2} \cdot \min \left\{r_{n}^{2 d+2} f_{n}^{2}\left((\delta+\varepsilon) r_{n}\right), \frac{f_{n}^{2}\left(R_{c}+(\delta+\varepsilon) r_{n}\right)}{(\log n)^{2 d /(d-1)}}\right\} ;
$$

(ii) If $\alpha \varepsilon^{d}>(d+3)^{d / 2}$, then w.h.p.

$$
\bar{\lambda}(t) \leq 1-c n^{2} f_{n}^{2}\left((\delta+\varepsilon) r_{n}\right) \min \left\{r_{n}^{2 d+2}, 1\right\},
$$

where $c$ is a positive constant depending on $\alpha, \varepsilon$ and $d$.

The proof of Lemma 5.3 is put in Appendix 6.

\subsection{Proofs of Theorem 3.1 and Corollary 3.4}

Proof of Theorem 3.1 By the fact that the row sum of $P(t)$ is 1 , we see that $P(t) \bar{V}=\bar{V}$. By (3), we have

$$
V(k+1)=P(k) P(k-1) \cdots P(0) V(0)=P(k) P(k-1) \cdots P(0)(V(0)-\bar{V})+\bar{V} .
$$

Using (26) and Corollary 1 in [11], we obtain that

$$
\|V(k)-\bar{V}\|_{F} \leq\|V(0)-\bar{V}\|_{F} \prod_{i=0}^{k-1} \bar{\lambda}(i) .
$$

Denote

$$
\lambda^{*}= \begin{cases}1-c n^{2} \min \left\{r_{n}^{2 d+2} f_{n}^{2}\left((\delta+\varepsilon) r_{n}\right), \frac{f_{n}^{2}\left(R_{c}+(\delta+\varepsilon) r_{n}\right)}{\left.(\log n)^{2 d /(d-1)}\right\},}\right. & \text { if } \alpha \varepsilon^{d} \leq(d+3)^{d / 2}, \\ 1-c n^{2} f_{n}^{2}\left((\delta+\varepsilon) r_{n}\right) \min \left\{r_{n}^{2 d+2}, 1\right\}, & \text { else, }\end{cases}
$$

where $c$ is the same constant appearing in Theorem 5.3. Take

$$
k^{*}=\left\lceil\log _{\frac{1}{\lambda^{*}}}\left(\frac{\|V(0)-\bar{V}\|_{F}}{\|V(0)-\bar{V}\|_{\max }}\right)\right\rceil .
$$

It is easy to see that $k^{*}$ satisfies $k^{*} \leq \frac{2}{1-\lambda^{*}} \log \left(\frac{\|V(0)-\bar{V}\|_{F}}{\|V(0)-\bar{V}\|_{\max }}\right)$. By the condition of the theorem, we have

$$
\|V(0)-\bar{V}\|_{\max }\left(\log \left(\frac{\|V(0)-\bar{V}\|_{F}}{\|V(0)-\bar{V}\|_{\max }}\right)+1\right) \leq \frac{\left(1-\lambda^{*}\right) \delta r_{n}}{4 \sqrt{d}}
$$

then

$$
2\|V(0)-\bar{V}\|_{\max }\left(\sqrt{d} k^{*}+\frac{1}{1-\lambda^{*}}\right) \leq \delta r_{n} .
$$

We assert that if (29) holds, then we have w.h.p.

$$
\max _{1 \leq i, j \leq n} d_{i j}(k) \leq \delta r_{n}, \quad \forall k \geq 0
$$


where $d_{i j}(k)=\left\|X_{i}(k)-X_{j}(k)-X_{i}(0)+X_{j}(0)\right\|$.

We prove the aforementioned assertion by induction. It is clear that (30) holds for $k=0$. We assume that the assertion holds for $0<s \leq k$, that is, w.h.p.

$$
\max _{i, j} d_{i j}(s) \leq \delta r_{n}, \quad \forall 0 \leq s \leq k .
$$

By Lemmas 5.1 and 5.2, the matrix $P(s)(0 \leq s \leq k)$ is stochastic. Denote

$$
a(t):=\left(\sum_{j=1}^{d}\left(\max _{1 \leq i_{1}, i_{2} \leq n}\left|v_{i_{1}, j}(t)-v_{i_{2}, j}(t)\right|^{2}\right)\right)^{1 / 2} .
$$

It is clear that the sequence $a(t)$ is monotonically non-increasing, and thus

$$
a(t) \leq a(0) \leq 2 \sqrt{d}\|V(0)-\bar{V}\|_{\max } .
$$

Moreover, using Lemma 5.3, we have $\bar{\lambda}(s) \leq \lambda^{*}$ for $s \leq k$. By (27) and (31), we obtain that w.h.p.

$$
\begin{aligned}
\max _{i, j}\left\{d_{i j}(k+1)\right\} & =\max _{i, j}\left\{\left\|\sum_{s=0}^{k-1}\left(V_{i}(s)-V_{j}(s)\right)\right\|\right\} \\
& \leq k^{*} a(0)+2 \sum_{s=k^{*}}^{k}\left(\max _{i}\left\|V_{i}(s)-V_{0}\right\|\right) \leq k^{*} a(0)+2 \sum_{s=k^{*}}^{k}\|V(s)-\bar{V}\|_{F} \\
& \leq k^{*} 2 \sqrt{d}\|V(0)-\bar{V}\|_{\max }+2\|V(0)-\bar{V}\|_{F} \sum_{s=k^{*}}^{k}\left(\lambda^{*}\right)^{s} \\
& \leq 2\|V(0)-\bar{V}\|_{\max }\left(\sqrt{d} k^{*}+\frac{1}{1-\lambda^{*}}\right) \leq \delta r_{n} .
\end{aligned}
$$

The assertion holds for $s=k+1$. By induction, the assertion holds for all $s \geq 0$.

By (27), (30) and Lemma 5.3, w.h.p.

$$
\lim _{k \rightarrow \infty}\|(V(k)-\bar{V})\|_{F} \leq\|V(0)-\bar{V}\|_{F} \cdot \lim _{k \rightarrow \infty}\left(\lambda^{*}\right)^{k}=0
$$

holds for all large $n$. Substituting the value of $\lambda^{*}$ into (28) yields our result.

Proof of Corollary 3.4 By Proposition 4.1, we see that there exists a constant $c^{\prime}=$ $c^{\prime}\left(d, \alpha, c_{0}\right)>0$, such that for any $\delta \in(0,1)$, we have $\bar{k}_{n, \delta} \leq\left(1+c^{\prime} \delta\right) \bar{k}_{n}$. Take $\delta=\frac{\varepsilon}{2 c^{\prime}}$, we have $\bar{k}_{n, \delta} \leq\left(1+\frac{\varepsilon}{2}\right) \bar{k}_{n}<1-\frac{\varepsilon}{2}$. Let $c_{1}=1+\frac{1}{2 c^{\prime}}$, the result follows by the same argument as that of Theorem 3.1.

\subsection{Proof of Theorem 3.8}

(i) Set $M_{n}:=\left\lfloor 1 /\left(2 R_{c}\right)\right\rfloor-1$, where $R_{c}=\sqrt[d]{\frac{2^{d-1} \log n}{d \pi_{d} n}}$, and $\lfloor x\rfloor$ denotes the largest integer no bigger than $x$. For any integer $k \in\left[0, M_{n}\right]$, define the point $x_{k}:=\left((2 k+1) R_{c}, 0, \cdots, 0\right) \in[0,1]^{d}$ and set the event

$$
A_{k}:=\left\{\mathcal{X}_{n} \cap B\left(x_{k} ; n^{-\frac{1}{d}}\right) \neq \emptyset, \mathcal{X}_{n} \cap\left[B\left(x_{k} ; r_{n}+n^{-\frac{1}{d}}\right) \backslash B\left(x_{k} ; n^{-\frac{1}{d}}\right)\right]=\emptyset\right\} .
$$


Let $B(x ; r)$ denotes the $d$-dimensional ball centered at $x$ with the radius $r$. By the similar analysis as that of the equation (4.13) in [27], we have for large $n$

$$
\begin{aligned}
& P\left(\bigcup_{0 \leq k \leq M_{n}} A_{k}\right)>1-2 \mathrm{e}^{-n^{1 / 4}}- {\left[1-\left(1-\exp \left\{-\frac{\left(n-n^{\frac{3}{4}}\right) \pi_{d}}{2^{d-1} n}\right\}\right)\right.} \\
&\left.\cdot \exp \left\{-\frac{\left(n+n^{\frac{3}{4}}\right) \pi_{d}\left[\left(r_{n}+n^{-\frac{1}{d}}\right)^{d}-\frac{1}{n}\right]}{2^{d-1}}\right\}\right]^{M_{n}+1} \\
&>1-2 \mathrm{e}^{-n^{1 / 4}}-\left[1-\frac{\pi_{d}}{2^{d}} \exp \left\{-\frac{\pi_{d} \alpha^{\prime} \log n}{2^{d-1}}\right\}\right]^{M_{n}+1} \\
&>1-2 \mathrm{e}^{-n^{1 / 4}}-\exp \left\{-n^{-\frac{\pi_{d} \alpha^{\prime \prime}}{2^{d-1}}+\frac{1}{d}}\right\}
\end{aligned}
$$

where the constants $\alpha^{\prime}, \alpha^{\prime \prime}$ satisfy $\alpha<\alpha^{\prime}<\alpha^{\prime \prime}<\frac{2^{d-1}}{d \pi_{d}}$. So, w.h.p. there must exist an integer $k \in\left[0, M_{n}\right]$ such that $A_{k}$ happens. Without loss of generality we assume $A_{0}$ happens, then there exist some agents lying in $B\left(x_{0} ; n^{-1 / d}\right)$ which have no neighbor in $[0,1]^{d} \backslash B\left(x_{0} ; n^{-1 / d}\right)$ at the initial time. Taking their initial velocities to be $(0,-v, \cdots,-v)$ and the other agents' initial velocities to be $(0, v, \cdots, v)$ for any $v>0$, we see that the system (3) cannot achieve flocking w.h.p.

(ii) Let $\widetilde{i}$ be the index of the agent whose position satisfies $\left\|X_{\widetilde{i}}(0)\right\| \leq\left\|X_{i}(0)\right\|$ for any $1 \leq i \leq n$. Then, for any constant $\varepsilon>0$ we have

$$
\begin{aligned}
P\left(\left\|X_{\widetilde{i}}(0)\right\|>2\left(\frac{\varepsilon \log n}{\pi_{d} n}\right)^{1 / d}\right) & =P\left(\bigcap_{i=1}^{n}\left\{\left\|X_{i}(0)\right\|>2\left(\frac{\varepsilon \log n}{\pi_{d} n}\right)^{1 / d}\right\}\right) \\
& =\left(1-\frac{\varepsilon \log n}{n}\right)^{n}=n^{-\varepsilon}(1+o(1))
\end{aligned}
$$

which indicates that $\left\|X_{\widetilde{i}}(0)\right\| \leq 2\left(\frac{\varepsilon \log n}{\pi_{d} n}\right)^{1 / d}$ holds w.h.p. For some constant $v_{0}$, we set $V_{\widetilde{i}}(0)=$ $\left(-\frac{v_{0}}{\sqrt{d}}, \cdots,-\frac{v_{0}}{\sqrt{d}}\right)^{\mathrm{T}}$, and $V_{i}(0)=-V_{\tilde{i}}(0)$ for $i \neq \widetilde{i}$.

If $\bar{k}_{n}=\Theta(1)$, we take $v_{0}=2^{-d-1} \bar{k}_{n} r_{n}$. By (3), w.h.p. all elements of $X_{\tilde{i}}(1)=X_{\tilde{i}}(0)+V_{\tilde{i}}(0)$ are less than 0 , and $\left\|X_{\widetilde{i}}(1)-X_{i}(1)\right\| \geq 2^{-d} \bar{k}_{n} r_{n}$ holds for $i \neq \widetilde{i}$. By Lemma 5.2 , it is easy to see that there exists a small constant $\varepsilon>0$ such that

$$
d_{\widetilde{i}}(1)=\sum_{i \neq \widetilde{i}} f_{n}\left(\left\|X_{\widetilde{i}}(1)-X_{i}(1)\right\|\right) \leq \frac{(1-\varepsilon) \bar{k}_{n}}{2^{d}} \quad \text { w.h.p.. }
$$

Thus, for $i \neq \widetilde{i}$,

$$
\begin{aligned}
& \left\|X_{\widetilde{i}}(2)-X_{i}(2)\right\| \\
= & \left\|X_{\widetilde{i}}(1)+\left[1-2 d_{\widetilde{i}}(1)\right] V_{\widetilde{i}}(0)-X_{i}(1)+\left[1-2 f_{n}\left(\left\|X_{\widetilde{i}}(1)-X_{i}(1)\right\|\right)\right] V_{\tilde{i}}(0)\right\| \\
\geq & 2^{-d} \bar{k}_{n} r_{n}\left(1+\left[1-\left(1-\frac{\varepsilon}{2}\right) \frac{\bar{k}_{n}}{2^{d}}\right]\right) \quad \text { w.h.p. }
\end{aligned}
$$


Repeating the above process we have for all $t>1$,

$$
\begin{aligned}
& \left\|X_{\widetilde{i}}(t)-X_{i}(t)\right\| \\
\geq & 2^{-d} \bar{k}_{n} r_{n}\left(1+\left[1-\left(1-\frac{\varepsilon}{2}\right) \frac{\bar{k}_{n}}{2^{d}}\right]+\cdots+\left[1-\left(1-\frac{\varepsilon}{2}\right) \frac{\bar{k}_{n}}{2^{d}}\right]^{t-1}\right) \quad \text { w.h.p. }
\end{aligned}
$$

Therefore, there exists a time instant $T$ such that w.h.p. for all $i \neq \widetilde{i}$ : (i) $\left\|X_{\widetilde{i}}(T)-X_{i}(T)\right\| \geq r_{n}$; (ii) all elements of $V_{\hat{i}}(T)$ are negative; (iii) all elements of $V_{i}(T)$ are positive. For such a case, the system cannot reach flocking.

If $\bar{k}_{n}=o(1)$, we take $v_{0}=\frac{1}{2} \bar{k}_{n} r_{n}$. Similarly we see that there exists a constant $\varepsilon>0$ such that w.h.p. $d_{\tilde{i}}(1) \leq(1-\varepsilon) \bar{k}_{n}$, and

$$
\left\|X_{\tilde{i}}(t)-X_{i}(t)\right\| \geq \bar{k}_{n} r_{n}\left(1+\left[1-\left(1-\frac{\varepsilon}{2}\right) \bar{k}_{n}\right]+\cdots+\left[1-\left(1-\frac{\varepsilon}{2}\right) \bar{k}_{n}\right]^{t-1}\right)
$$

holds for $t>1$. Thus, the system cannot reach flocking.

\section{Concluding Remarks}

A fundamental issue in the investigation of multi-agent systems is how the local interactions affect the collective behavior of the overall systems. This paper studied a discrete-time nonlinear multi-agent system, where the nonlinear interaction function decays according to the distance between agents. By applying large deviations techniques to estimate the essential spectral gap of average matrices whose elements are determined by the nonlinear function and the distance between agents, we provide sufficient conditions and necessary conditions for the flocking behavior. Some interesting problems deserve to be further investigated, for example, how to obtain the critical value of $v$ for $v$-flocking of our multi-agent model, and how to analyze the flocking behavior of the continuous-time multi-agent models.

\section{References}

[1] Toner J and Tu Y, Flocks, herds, and schools: A quantitative theory of flocking, Phys. Rev. E, 1998, 58(4): 4828-4858.

[2] Reynolds C, Flocks, herds, and schools: A distributed behavioral model, Computer Graphics, 1987, 21: 25-34.

[3] Vicsek T, Czirók A, Jacob E B, et al., Novel type of phase transition in a system of self-driven particles, Phys. Rev. Lett., 1995, 75: 1226-1229.

[4] Buhl J, Sumpter D J T, Couzin I D, et al., From disorder to order in marching locusts, Science, 2006, 312(5778): 1402-1406.

[5] Chazelle B, The convergence of bird flocking, Journal of the ACM, 2014, 61(4): 1-35.

[6] Olfati-Saber R, Flocking for multi-agent dynamic systems: Algorithms and theory, IEEE Trans. Autom. Control, 2006, 51(3): 401-420. 
[7] Jadbabaie A, Lin J, and Morse A S, Coordination of groups of mobile autonomous agents using nearest neighbor rules, IEEE Trans. Autom. Control, 2003, 48(9): 988-1001.

[8] Savkin A V, Coordinated collective motion of groups of autonomous mobile robots: Analysis of Vicsek's model, IEEE Trans. Autom. Control, 2004, 39: 981-983.

[9] Li Q and Jiang Z P, Global analysis of multi-agent systems based on Vicsek's model, IEEE Trans. Auto. Control, 2009, 54(12): 2876-2881.

[10] Chen G, Small noise may diversify collective motion in Vicsek model, IEEE Trans. Auto. Control, 2017, 62(2): 636-651.

[11] Cucker F and Smale S, Emergent behavior in flocks, IEEE Trans. Autom. Control, 2007, 52(5): 852-862.

[12] Cucker F and Mordecki E, Flocking in noisy environments, J. Math. Pures Appl., 2008, 89: $278-296$.

[13] Cucker F and Dong J G, A general collision-avoiding flocking framework, IEEE Trans. Autom. Control, 2011, 56: 1124-1129.

[14] Peszek J, Existence of piecewise weak solutions of a discrete Cucker-Smale's flocking model with a singular communication weight, Journal of Differential Equations, 2014, 257(8): 2900-2925.

[15] Carrillo J A, Fornasier M, Rosado J, et al., Asymptotic flocking dynamics for the kinetic CuckerSmale model, SIAM J. Math. Anal., 2010, 42(1): 218-236.

[16] Ahn S M and Ha S, Stochastic flocking dynamics of the Cucker-Smale model with multiplicative white noises, Journal of Mathematical Physics, 2010, 51(10): 1634-1642.

[17] Shen J, Cucker-Smale flocking under hierarchical leadership, SIAM J. Appl. Math., 2007, 68(3): 694-719.

[18] Park J, Kim H J, and Ha S Y, Cucker-Smale flocking with inter-particle bonding forces, IEEE Trans. Auto. Control, 2010, 55(11): 2617-2623.

[19] Ha S Y, Ha T, and Kim J H, Emergent behavior of a Cucker-Smale type particle model with nonlinear velocity couplings, IEEE Trans. Autom. Control, 2010, 55(7): 1679-1683.

[20] Ha S Y, Jeong J, Noh S E, et al., Emergent dynamics of Cucker-Smale flocking particles in a random environment, Journal of Differential Equations, 2017, 262(3): 2554-2591.

[21] Rosenthal S B, Twomey C R, Hartnett A T, et al., Revealing the hidden networks of interaction in mobile animal groups allows prediction of complex behavioral contagion, P. Natl. Acad. Sci. USA, 2015, 112(15): 4690-4695.

[22] Rieu J P, Upadhyaya A, Glazier J A, et al., Diffusion and deformations of single Hydra cells in cellular aggregates, Biophys. J., 2000, 79(4): 1903-1914.

[23] Rieu J P, Kataoka N, and Sawada Y, Quantitative analysis of cell motion during sorting in two-dimensional aggregates of dissociated Hydra cells, Phys. Rev. E, 1998, 57(1): 924-931.

[24] Martin S, Girard A, Fazeli A, et al., Multiagent flocking under general communication rule, IEEE Transactions on Control of Network Systems, 2014, 1(2): 155-166.

[25] Tang G G and Guo L, Convergence of a class of multi-agent systems in probabilistic framework, Journal of Systems Science and Complexity, 2007, 20(2): 173-197.

[26] Liu Z X and Guo L, Synchronization of multi-agent systems without connectivity assumption, Automatica, 2009, 45: 2744-2753.

[27] Chen G, Liu Z X, and Guo L, The smallest possible interaction radius for synchronization of self-propelled particles, SIAM Rev., 2014, 56(3): 499-521.

[28] Penrose M D, Random Geometric Graphs, Oxford University Press, Oxford, UK, 2003. 
[29] Gupta P and Kumar P R, The capacity of wireless networks, IEEE Trans. Inform. Theory, 2000, 46: 388-404.

[30] Dembo A and Zeitouni O, Large Deviations Techniques and Applications, 2nd Edition, Springer, New York, 1998.

[31] Gupta P and Kumar P R, Critical power for asymptotic connectivity in wireless networks, Stochastic Analysis, Control, Optimization and Applications, Birkhäuser Boston, Boston, MA, 1999, 547-566.

[32] Penrose M D, The longest edge of the random minimal spanning tree, Ann. Appl. Probab., 1997, 7(2): $340-361$.

[33] Diaconis P and Strook D, Geometric bounds for eigenvalues of Markov chains, Ann. Appl. Probab., 1991, 1: 36-61.

\section{Appendix}

\section{Proof of Lemma 2.1}

The lemma holds for $d=2$, see [31] and [32]. In the following, we prove the lemma for $d \geq 3$.

For $\alpha>\frac{2^{d-1}}{d \pi_{d}}$, by Theorem 7.14 in [28], we see that the minimum degree of $G\left(\mathcal{X}_{n} ; r_{n}\right)$ is equal to $\Theta\left(n r_{n}^{d}\right)$ w.h.p., which indicates that there is no isolated vertex in $G\left(\mathcal{X}_{n} ; r_{n}\right)$ w.h.p. Following the proof idea of Theorem 3.1 in [31], we see that for $d \geq 3$, the probability that $G\left(\mathcal{X}_{n} ; r_{n}\right)$ is not connected has the same order as the probability that the graph $G\left(\mathcal{X}_{n} ; r_{n}\right)$ has isolated vertices. Thus, the graph $G\left(\mathcal{X}_{n} ; r_{n}\right)$ is connected w.h.p.

For $\alpha<\frac{2^{d-1}}{d \pi_{d}}$, by the proof of Theorem 3.8 (i) we see that for some vertex $k$, w.h.p. there exist some vertices lying in $B\left(x_{k} ; n^{-1 / d}\right)$ which do not have neighbors in $[0,1]^{d} \backslash B\left(x_{k} ; n^{-1 / d}\right)$. Thus, the graph $G\left(\mathcal{X}_{n} ; r_{n}\right)$ is not connected w.h.p. This completes the proof of the lemma.

\section{Proof of Proposition 4.1}

(i) By the definition of $\xi_{n}$, we have

$$
\begin{aligned}
E\left[\mathrm{e}^{\bar{\theta}_{n} \xi_{n}}\right] & =1-\pi_{d} r_{n}^{d}+d \pi_{d} \int_{0}^{r_{n}} \mathrm{e}^{\bar{\theta}_{n} f_{n}(x)} x^{d-1} d x \\
& =1-\pi_{d} r_{n}^{d}+d \pi_{d} r_{n}^{d} \int_{0}^{1} \mathrm{e}^{\bar{\theta}_{n} f_{n}\left(r_{n} y\right)} y^{d-1} d y \\
E\left[\xi_{n} \mathrm{e}^{\bar{\theta}_{n} \xi_{n}}\right] & =d \pi_{d} r_{n}^{d} \int_{0}^{1} f_{n}\left(r_{n} y\right) \mathrm{e}^{\bar{\theta}_{n} f_{n}\left(r_{n} y\right)} y^{d-1} d y
\end{aligned}
$$

Substituting the above two equations into (16) and using the assumption $\bar{\theta}_{n}=O\left(1 / f_{n}(0)\right)$, we have

$$
\begin{aligned}
& n \bar{\theta}_{n} d \pi_{d} r_{n}^{d} \int_{0}^{1} f_{n}\left(r_{n} y\right) \mathrm{e}^{\bar{\theta}_{n} f_{n}\left(r_{n} y\right)} y^{d-1} d y \\
= & \left(\log n+n\left(-\pi_{d} r_{n}^{d}+d \pi_{d} r_{n}^{d} \int_{0}^{1} \mathrm{e}^{\bar{\theta}_{n} f_{n}\left(r_{n} y\right)} y^{d-1} d y\right)\right)\left(1 \pm O\left(r_{n}^{d}\right)\right),
\end{aligned}
$$


which indicates that

$$
\int_{0}^{1}\left(\bar{\theta}_{n} f_{n}\left(r_{n} y\right)-1\right) \mathrm{e}^{\bar{\theta}_{n} f_{n}\left(r_{n} y\right)} y^{d-1} d y=\left(\frac{\log n}{d \pi_{d} n r_{n}^{d}}-\frac{1}{d}\right)\left(1 \pm O\left(r_{n}^{d}\right)\right) \pm O\left(r_{n}^{d}\right) .
$$

Let $\theta_{n}^{*}$ denote a solution of the following equation

$$
\int_{0}^{1}\left(\theta_{n} f_{n}\left(r_{n} y\right)-1\right) \mathrm{e}^{\theta_{n} f_{n}\left(r_{n} y\right)} y^{d-1} d y=\frac{\log n}{d \pi_{d} n r_{n}^{d}}-\frac{1}{d}
$$

with respect to $\theta_{n}$. Note that if $\theta_{n}=0$ then

$$
\text { the left side of }(34)<\text { the right side of }(34) \text {, }
$$

and if $\theta_{n}>\log n /\left(d \pi_{d} c_{0} n r_{n}^{d} f_{n}(0)\right)$, then by (10),

the left side of (34)

$$
\begin{aligned}
& =\frac{1}{d}\left[\int_{0}^{1} \theta_{n} f_{n}\left(r_{n} z^{1 / d}\right) \mathrm{e}^{\theta_{n} f_{n}\left(r_{n} z^{1 / d}\right)} d z-\int_{0}^{1} \mathrm{e}^{\theta_{n} f_{n}\left(r_{n} z^{1 / d}\right)} d z\right] \\
& \geq \frac{1}{d}\left[\int_{0}^{1} \theta_{n} f_{n}\left(r_{n} z^{1 / d}\right) d z \int_{0}^{1} \mathrm{e}^{\theta_{n} f_{n}\left(r_{n} z^{1 / d}\right)} d z-\int_{0}^{1} \mathrm{e}^{\theta_{n} f_{n}\left(r_{n} z^{1 / d}\right)} d z\right] \\
& \geq \frac{1}{d}\left(\theta_{n} d c_{0} f_{n}(0)-1\right) \int_{0}^{1} \mathrm{e}^{\theta_{n} f_{n}\left(r_{n} z^{1 / d}\right)} d z \\
& >\text { the right side of }(34),
\end{aligned}
$$

where the following inequality is used

$$
\frac{a_{1} b_{1}+\cdots+a_{m} b_{m}}{m}-\frac{a_{1}+\cdots a_{m}}{m} \cdot \frac{b_{1}+\cdots+b_{m}}{m}=\frac{1}{m^{2}} \sum_{1 \leq i<j \leq m}\left(a_{i}-a_{j}\right)\left(b_{i}-b_{j}\right) \geq 0
$$

with $\left\{a_{i}, 1 \leq i \leq m\right\}$ and $\left\{b_{i}, 1 \leq i \leq m\right\}$ being two real number sequences satisfying $a_{1} \geq a_{2} \geq$ $\cdots \geq a_{m}$ and $b_{1} \geq b_{2} \geq \cdots \geq b_{m}$. Hence, by (35) and (36) we have

$$
0<\theta_{n}^{*}<\frac{\log n}{d \pi_{d} c_{0} n r_{n}^{d} f_{n}(0)}
$$

By (37) and (5), we have $\theta_{n}^{*}=O\left(1 / f_{n}(0)\right)$. Substituting this into (33), we see that the equation (16) has a solution near to $\theta_{n}^{*}$. By the uniqueness of the solution of (16) we obtain that $\bar{\theta}_{n}=\theta_{n}^{*}(1+o(1))=O\left(1 / f_{n}(0)\right)$. Moreover, by the first equation of (16), we have

$$
\bar{k}_{n}=n d \pi_{d} r_{n}^{d} \int_{0}^{1} f_{n}\left(r_{n} y\right) \mathrm{e}^{\theta_{n}^{*} f_{n}\left(r_{n} y\right)} y^{d-1} d y(1+o(1))=\Theta\left(n r_{n}^{d} f_{n}(0)\right) .
$$

(ii) Similar to the analysis of (i), we have

$$
\begin{aligned}
& E\left[\mathrm{e}^{\theta \xi_{n, \delta}}\right]=1-\pi_{d} r_{n}^{d}(1+\delta)^{d}+d \pi_{d} r_{n}^{d}(1+\delta)^{d} \int_{0}^{1} \mathrm{e}^{\theta f_{n, \delta}\left[(1+\delta) r_{n} y\right]} y^{d-1} d y, \\
& E\left[\xi_{n, \delta} \mathrm{e}^{\theta \xi_{n, \delta}}\right]=d \pi_{d} r_{n}^{d}(1+\delta)^{d} \int_{0}^{1} f_{n, \delta}\left[(1+\delta) r_{n} y\right] \mathrm{e}^{\theta f_{n, \delta}\left[(1+\delta) r_{n} y\right]} y^{d-1} d y .
\end{aligned}
$$


Now, we consider the solution of the following equation,

$$
\int_{0}^{1}\left(\theta f_{n, \delta}\left[(1+\delta) r_{n} y\right]-1\right) \mathrm{e}^{\theta f_{n, \delta}\left[(1+\delta) r_{n} y\right]} y^{d-1} d y=\frac{\log n}{d \pi_{d} n r_{n}^{d}(1+\delta)^{d}}-\frac{1}{d} .
$$

Define $g(y):=f_{n, \delta}\left[(1+\delta) r_{n} y\right]-f_{n}\left(r_{n} y\right)$. It is clear that $g(y) \geq 0$ for $y \leq 1$. Note that

$$
\begin{aligned}
& \int_{0}^{1}\left(\theta_{n}^{*} f_{n, \delta}\left[(1+\delta) r_{n} y\right]-1\right) \mathrm{e}^{\theta_{n}^{*} f_{n, \delta}\left[(1+\delta) r_{n} y\right]} y^{d-1} d y-\frac{\log n}{d \pi_{d} n r_{n}^{d}(1+\delta)^{d}}+\frac{1}{d} \\
& >\int_{0}^{1}\left\{\theta_{n}^{*}\left[f_{n}\left(r_{n} y\right)+g(y)\right]-1\right\} \mathrm{e}^{\theta_{n}^{*} f_{n}\left(r_{n} y\right)} \mathrm{e}^{\theta_{n}^{*} g(y)} y^{d-1} d y \\
& -\int_{0}^{1}\left(\theta_{n}^{*} f_{n}\left(r_{n} y\right)-1\right) \mathrm{e}^{\theta_{n}^{*} f_{n}\left(r_{n} y\right)} y^{d-1} d y \\
& =\int_{0}^{1}\left(\theta_{n}^{*} f_{n}\left(r_{n} y\right)-1\right) \mathrm{e}^{\theta_{n}^{*} f_{n}\left(r_{n} y\right)}\left(\mathrm{e}^{\theta_{n}^{*} g(y)}-1\right) y^{d-1} d y \\
& +\int_{0}^{1} \theta_{n}^{*} g(y) \mathrm{e}^{\theta_{n}^{*} f_{n}\left(r_{n} y\right)} \mathrm{e}^{\theta_{n}^{*} g(y)} y^{d-1} d y \\
& >\int_{0}^{1} \mathrm{e}^{\theta_{n}^{*} f_{n}\left(r_{n} y\right)}\left[\theta_{n}^{*} g(y) \mathrm{e}^{\theta_{n}^{*} g(y)}-\mathrm{e}^{\theta_{n}^{*} g(y)}+1\right] y^{d-1} d y \\
& =\int_{0}^{1} \mathrm{e}^{\theta_{n}^{*} f_{n}\left(r_{n} y\right)}\left[\sum_{i=2}^{\infty} \frac{i-1}{i !}\left(\theta_{n}^{*} g(y)\right)^{i}\right] y^{d-1} d y>0 .
\end{aligned}
$$

If $\theta=0$, then

$$
\text { the left side of }(39)<\text { the right side of }(39) \text {. }
$$

Thus, the solution of equation (39) satisfies $\theta_{n, \delta}^{*} \in\left(0, \theta_{n}^{*}\right)$. With a similar argument, we have $\bar{\theta}_{n, \delta}=\theta_{n, \delta}^{*}(1+o(1))$. For any $\delta>0$, similar to (38) we have

$$
\begin{aligned}
\frac{\bar{k}_{n, \delta}}{n d \pi_{d} r_{n}^{d}} & =(1+\delta)^{d} \int_{0}^{1} f_{n, \delta}\left[(1+\delta) r_{n} y\right] \mathrm{e}^{\theta_{n, \delta}^{*} f_{n, \delta}\left[(1+\delta) r_{n} y\right]} y^{d-1} d y(1+o(1)) \\
& =\int_{0}^{1+\delta} f_{n, \delta}\left(r_{n} z\right) \mathrm{e}^{\theta_{n, \delta}^{*} f_{n, \delta}\left(r_{n} z\right)} z^{d-1} d z(1+o(1)) .
\end{aligned}
$$

By the definition of $f_{n, \delta}$ and the fact that $\theta_{n, \delta}^{*}<\theta_{n}^{*}$ we have

$$
\begin{aligned}
& \int_{0}^{1+\delta} f_{n, \delta}\left(r_{n} z\right) \mathrm{e}^{\theta_{n, \delta}^{*} f_{n, \delta}\left(r_{n} z\right)} z^{d-1} d z \\
= & \int_{0}^{\delta} f_{n}(0) \mathrm{e}^{\theta_{n, \delta}^{*} f_{n}(0)} z^{d-1} d z+\int_{0}^{1} f_{n}\left(r_{n} z\right) \mathrm{e}^{\theta_{n, \delta}^{*} f_{n}\left(r_{n} z\right)}(z+\delta)^{d-1} d z \\
< & \frac{\delta^{d} f_{n}(0) \mathrm{e}^{\theta_{n}^{*} f_{n}(0)}}{d}+\sum_{i=0}^{d-1}\left(\begin{array}{c}
d-1 \\
i
\end{array}\right) \delta^{i} \int_{0}^{1} f_{n}\left(r_{n} z\right) \mathrm{e}^{\theta_{n}^{*} f_{n}\left(r_{n} z\right)} z^{d-1-i} d z \\
\leq & f_{n}(0) \mathrm{e}^{\theta_{n}^{*} f_{n}(0)}\left(\frac{\delta^{d}}{d}+\sum_{i=1}^{d-1}\left(\begin{array}{c}
d-1 \\
i
\end{array}\right) \frac{\delta^{i}}{d-i}\right)+\int_{0}^{1} f_{n}\left(r_{n} z\right) \mathrm{e}^{\theta_{n}^{*} f_{n}\left(r_{n} z\right)} z^{d-1} d z \\
= & (1+O(\delta)) \int_{0}^{1} f_{n}\left(r_{n} z\right) \mathrm{e}^{\theta_{n}^{*} f_{n}\left(r_{n} z\right)} z^{d-1} d z,
\end{aligned}
$$


where the condition (10) and the fact $\theta_{n}^{*}=O\left(1 / f_{n}(0)\right)$ are used in the last equation. Combining this with (40) and (38) our result follows.

(iii) Immediate from (37) and (38).

\section{Proof of Lemma 5.2}

Before the proof of Lemma 5.2, we need to introduce some notations. For any $n \in \mathbb{N}$, define $\rho(n):=\left\lfloor n-2 n^{3 / 4}\right\rfloor$ and

$$
\widehat{I}_{n, \delta}(x):=\sup _{\theta>0}\left\{\theta x-(\rho(n)-1) \log \left(E\left[\mathrm{e}^{\theta \xi_{n, \delta}}\right]\right)\right\} .
$$

Take $\widehat{k}_{n, \delta}>(\rho(n)-1) E\left[\xi_{n, \delta}\right]$ such that $\widehat{I}_{n, \delta}\left(\widehat{k}_{n, \delta}\right)=\log n$. Denote $\widetilde{f}_{n}=\widetilde{f}_{n, 0}, \widetilde{\xi}_{n}=\widetilde{\xi}_{n, 0}$, $\widetilde{I}_{n}=\widetilde{I}_{n, 0}$ and $\widehat{k}_{n}=\widehat{k}_{n, 0}$.

Lemma 6.1 For $\widehat{k}_{n}$ and $\bar{k}_{n}$, we have $\lim _{n \rightarrow \infty}\left(\widehat{k}_{n} / \bar{k}_{n}\right)=1$.

Proof First, by

$$
\widehat{I}_{n}\left(\bar{k}_{n}\right) \geq I_{n}\left(\bar{k}_{n}\right)=\log n=\widehat{I}_{n}\left(\widehat{k}_{n}\right),
$$

the inequality $\bar{k}_{n} \geq \widehat{k}_{n}$ can be derived.

Assume that there exists a constant $\varepsilon>0$ such that $\bar{k}_{n} \geq(1+\varepsilon) \widehat{k}_{n}$. Then for large $n$,

$$
\begin{aligned}
I_{n}\left(\bar{k}_{n}\right) & \geq I_{n}\left((1+\varepsilon) \widehat{k}_{n}\right) \\
& =\sup _{\theta>0}\left\{\theta(1+\varepsilon) \widehat{k}_{n}-(n-1) \log \left(E\left[\mathrm{e}^{\theta \xi_{n}}\right]\right)\right\} \\
& \geq \frac{n-1}{\rho(n)-1} \sup _{\theta>0}\left\{\theta\left(1+\frac{\varepsilon}{2}\right) \widehat{k}_{n}-(\rho(n)-1) \log \left(E\left[\mathrm{e}^{\theta \xi_{n}}\right]\right)\right\} \\
& \geq \frac{n-1}{\rho(n)-1}\left(1+\frac{\varepsilon}{2}\right) \widehat{I}_{n}\left(\widehat{k}_{n}\right) \\
& >\log n=I_{n}\left(\bar{k}_{n}\right),
\end{aligned}
$$

which leads to contradiction. Our result yields.

Proof of Lemma 5.2 For simplicity of expressions, we consider the case of $\delta=0$, and it is easy to extend our results to the case of $\delta>0$.

Denote $d_{i}=d_{i}(n):=\sum_{1 \leq j \leq n, j \neq i} f_{n}\left(\left\|X_{i}(0)-X_{j}(0)\right\|\right)$. For $k_{n}>(n-1) E\left[\xi_{n}\right]$, by $(2.2 .12)$ in [30], we have

$$
\begin{aligned}
P\left(d_{i}>k_{n}\right) & \leq P\left(\sum_{1 \leq j \leq n, j \neq i} f_{n}\left(\left\|x_{0}-X_{j}(0)\right\|\right)>k_{n}\right) \\
& =P\left(\frac{1}{n-1} \sum_{1 \leq j \leq n, j \neq i} f_{n}\left(\left\|x_{0}-X_{j}(0)\right\|\right)>\frac{k_{n}}{n-1}\right) \\
& \leq \exp \left(-(n-1) \sup _{\theta>0}\left\{\frac{\theta k_{n}}{n-1}-\log \left(E\left[\mathrm{e}^{\theta \xi_{n}}\right]\right)\right\}\right)=\mathrm{e}^{-I_{n}\left(k_{n}\right)},
\end{aligned}
$$


where $I_{n}(\cdot)$ is defined by $(6)$. For any $\varepsilon>0$ we have

$$
\begin{aligned}
& I_{n}\left((1+\varepsilon) \bar{k}_{n}\right) \\
\geq & \bar{\theta}_{n} \bar{k}_{n}(1+\varepsilon)-(n-1) \log \left(E\left[\mathrm{e}^{\bar{\theta}_{n} \xi_{n}}\right]\right) \quad(\text { according to }(6)) \\
\geq & (1+\varepsilon)\left(\bar{\theta}_{n} \bar{k}_{n}-(n-1) \log \left(E\left[\mathrm{e}^{\bar{\theta}_{n} \xi_{n}}\right]\right)\right) \quad(\text { according to }(16)) \\
= & (1+\varepsilon) \log n .
\end{aligned}
$$

Combining (42) with (41), we obtain

$$
P\left(d_{i}>(1+\varepsilon) \bar{k}_{n}\right) \leq \mathrm{e}^{-I_{n}\left((1+\varepsilon) \bar{k}_{n}\right)} \leq \mathrm{e}^{-(1+\varepsilon) \log n}=n^{-1-\varepsilon} .
$$

Set

$$
\bar{F}_{n}:=\bigcup_{i=1}^{n} I_{\left\{d_{i}>(1+\varepsilon) \bar{k}_{n}\right\}} .
$$

By the Boole's inequality and (43), we have

$$
P\left(\bar{F}_{n}\right) \leq \sum_{i=1}^{n} P\left(d_{i}>(1+\varepsilon) \bar{k}_{n}\right) \leq n^{-\varepsilon} .
$$

Thus,

$$
P\left(\Delta_{n}>(1+\varepsilon) \bar{k}_{n}\right)=P\left(\bar{F}_{n}\right) \rightarrow 0 \quad \text { as } n \rightarrow \infty .
$$

The inequality (24) holds.

\section{Proof of Lemma 5.3}

The proof of Lemma 5.3 mainly uses the idea appearing in the proof of Theorem 4.3 of [27]. We first introduce some notations.

For any constant $\varepsilon \in(0,1)$, set $K_{n}=K_{n}(\varepsilon):=\left\lceil\frac{\sqrt{d+3}}{\varepsilon r_{n}}\right\rceil$, where $\lceil x\rceil$ is the smallest integer no less than $x$. The unit square $[0,1]^{d}$ is divided into $K_{n}^{d}$ equal small squares with the length of each side equal to $1 / K_{n}$. We denote these small squares as $S_{1}(n), S_{2}(n), \cdots, S_{K_{n}^{d}}(n)$. Denote $S_{i}=S_{i}(n)$ for $1 \leq i \leq K_{n}^{d}$. For each small square $S_{i}, 1 \leq i \leq K_{n}^{d}$, let $x_{i}$ denote its center point, and $z_{i}:=K_{n} x_{i}+\left(\frac{1}{2}, \frac{1}{2}, \cdots, \frac{1}{2}\right) \in \mathbb{Z}^{d}$.

Let $\|\cdot\|_{1}$ and $\|\cdot\|_{\infty}$ denote the $l_{1}$-norm and infinity norm respectively. For any $x, y \in \mathbb{Z}^{d}$, if $\|x-y\|_{1}=1$, then we say that $x$ and $y$ are adjacent, written as $x \sim y$. Given $A \subseteq \mathbb{Z}^{d}$, if for any $x, y \in A$, there exists a vertex sequence $x_{1}, x_{2}, \cdots, x_{n}$ in $A$ such that $x \sim x_{1}, x_{1} \sim x_{2}, x_{2} \sim$ $x_{3}, \cdots, x_{n} \sim y$, then we say $A$ is connected. Similarly, if $\|x-y\|_{\infty} \leq k, k \geq 1$, we say that $x$ and $y$ are $k$-adjacent, written as $x \sim_{k} y$. Given $A \subseteq \mathbb{Z}^{d}$, if for any $x, y \in A$, there exists a vertex sequence $x_{1}, x_{2}, \cdots, x_{n}$ in $A$ such that $x \sim_{k} x_{1}, x_{1} \sim_{k} x_{2}, x_{2} \sim_{k} x_{3}, \cdots, x_{n} \sim_{k} y$, then we say $A$ is $k$-connected. We see that for any $k \geq 1$ if $A$ is $k$-connected, then $A$ must be connected. In particular, a single vertex set $\{x\} \subset \mathbb{Z}^{d}$ is both connected and $k$-connected.

We define the lattice box $B_{\mathbb{Z}}\left(K_{n}\right)$ by $B_{\mathbb{Z}}\left(K_{n}\right):=\prod_{i=1}^{d}\left(\left[1, K_{n}\right] \cap \mathbb{Z}\right)$. It is clear that $B_{\mathbb{Z}}\left(K_{n}\right)$ is equal to the set $\left\{z_{i}: 1 \leq i \leq K_{n}^{d}\right\}$. For $A \subset B_{\mathbb{Z}}\left(K_{n}\right)$, we denote $A^{c}:=B_{\mathbb{Z}}\left(K_{n}\right) \backslash A$. 
Let $\partial A$ denote the internal vertex-boundary of $A$, that is, the set of vertex $z \in A$ such that $\left\{y \in A^{c}:\|z-y\|_{1}=1\right\}$ is non-empty.

For $\eta>0$, we use $P o(\eta)$ to denote the Poisson random variable with parameter $\eta$. Define a Poisson point process $\mathcal{P}_{\eta}$ as $\mathcal{P}_{\eta}:=\left\{Y_{1}, Y_{2}, \cdots, Y_{P o(\eta)}\right\}$, where $\left\{Y_{1}, Y_{2}, \cdots\right\}$ is the set of vertices independently and uniformly distributed in $[0,1]^{d}$ and $P o(\eta)$ is independent of $\left\{Y_{1}, Y_{2}, \cdots\right\}$, see Subsection 1.7 in [28]. For a Borel set $A \subseteq[0,1]^{d},\left|\mathcal{P}_{\eta} \cap A\right|$ is a Poisson random variable with parameter $\eta L e b(A)$, where $|\cdot|$ denotes the cardinality and $\operatorname{Leb}(\cdot)$ denotes the Lebesgue measure. For any two Borel sets $A_{1}, A_{2} \subseteq[0,1]^{d}$, if $\operatorname{Leb}\left(A_{1} \cap A_{2}\right)=0$, then the random variables $\left|\mathcal{P}_{\eta} \cap A_{1}\right|$ and $\left|\mathcal{P}_{\eta} \cap A_{2}\right|$ are mutually independent. Set $\eta(n):=n-n^{3 / 4}$, and let $\mathcal{P}_{\eta(n)}$ be a Poisson point process in $[0,1]^{d}$ with parameter $\eta(n)$. Then, $\mathcal{P}_{\eta(n)} \subseteq \mathcal{X}_{n}$ except when $\operatorname{Po}(\eta(n))>n$, and by Lemma 1.4 in [28] we obtain

$$
\mathcal{P}_{\eta(n)} \subseteq \mathcal{X}_{n} \quad \text { w.h.p. }
$$

For any set $A \subseteq B_{\mathbb{Z}}\left(K_{n}\right)$, define the function

$$
g_{1}(A):=\sum_{z_{i} \in A, z_{j} \in A^{c}, z_{i} \sim z_{j}}\left|\mathcal{P}_{\eta(n)} \cap S_{i}\right| \cdot\left|\mathcal{P}_{\eta(n)} \cap S_{j}\right| .
$$

For any $z_{i} \in B_{\mathbb{Z}}\left(K_{n}\right)$, we call $z_{i}$ open if $S_{i} \cap \mathcal{P}_{\eta(n)} \neq \emptyset$, and call $z_{i}$ closed otherwise. Let $\mathcal{O}_{n}$ denote the set of open vertices in $B_{\mathbb{Z}}\left(K_{n}\right)$, and let $\mathcal{C}_{n}$ denote the largest open clusters of $\mathcal{O}_{n}$.

Before proving Lemma 5.3, we provide some preliminary results, see the following Lemmas 6.2-6.7. Lemmas 6.2 and 6.4-6.7 are proved under the condition (5), and we will omit it to avoid repetition.

Lemma 6.2 There exists a constant $c=c(\varepsilon, \alpha, d)$ such that

$$
\max _{1 \leq i \leq K_{n}^{d}}\left|\mathcal{X}_{n} \cap S_{i}\right| \leq c \varepsilon^{d} n r_{n}^{d} \quad \text { w.h.p. }
$$

Proof It can be easily deduced from Lemma 4.1 of [27].

Lemma 6.3 (Lemma 9.9 in [28]) Let $\beta \in(0,1)$. If $A$ is a subset of $B_{\mathbb{Z}}\left(K_{n}\right)$ (not necessarily connected), with $|A| \leq \beta K_{n}^{d}$, then

$$
|\partial A| \geq(2 d)^{-1}\left(1-\beta^{1 / d}\right)|A|^{(d-1) / d} .
$$

Lemma 6.4 Suppose that $A \subset B_{\mathbb{Z}}\left(K_{n}\right)$ and the integer $k \geq 1$. Then for any $\beta \in(0,1)$, there exist constants $c=c(\alpha, \varepsilon, k, \beta, d)>0$ and $\gamma=\gamma(\alpha, \varepsilon, k, \beta)$ such that w.h.p.

$$
\inf _{\substack{\gamma(\log n)^{d /(d-1)} \leq|A| \leq \beta K_{n}^{d} \\ A \text { is } k \text {-connected }}} \frac{g_{1}(A)}{|A|}>\frac{c n^{2} r_{n}^{2 d}}{K_{n}} .
$$

Proof The lemma can be deduced by the similar method as that of Lemma 5.10 in [27] with a small modification, and we omit the proof details to save space. 
Lemma 6.5 If $\alpha \varepsilon^{d}>(d+3)^{d / 2}$ then w.h.p.

$$
\min _{1 \leq i \leq K_{n}^{d}}\left|S_{i} \cap \mathcal{P}_{\eta(n)}\right|>\frac{n}{K_{n}^{d}} H_{-}^{-1}\left(\frac{1}{2}+\frac{(d+3)^{d / 2}}{2 \alpha \varepsilon^{d}}\right) .
$$

Proof By Lemma 1.2 in [28] we obtain

$$
\begin{aligned}
& P\left(\bigcup_{i=1}^{K_{n}^{d}}\left\{\left|\mathcal{P}_{\eta(n)} \cap S_{i}\right| \leq \frac{n \beta}{K_{n}^{d}}\right\}\right) \\
\leq & K_{n}^{d} \exp \left(-\frac{n-n^{3 / 4}}{\left\lceil\frac{\sqrt{d+3}}{\varepsilon r_{n}}\right\rceil^{d}} H\left(\frac{n \beta}{n-n^{3 / 4}}\right)\right) \\
< & n \exp \left(-\frac{n-n^{3 / 4}}{\left\lceil\frac{\sqrt{d+3}}{\varepsilon r_{n}}\right\rceil^{d}}\left(\frac{1}{3}+\frac{2}{3 \alpha \varepsilon^{d}(d+3)^{-d / 2}}\right)\right) \rightarrow 0 \text { as } n \rightarrow \infty,
\end{aligned}
$$

which is followed by our result.

Lemma 6.6 Suppose that $\alpha \varepsilon^{d}>(d+3)^{d / 2}$ and $A \subset B_{\mathbb{Z}}\left(K_{n}\right)$. Then for any $\beta \in(0,1)$, there exists a constant $c=c(\alpha, d, \beta)>0$ such that w.h.p.

$$
\inf _{|A| \leq \beta K_{n}^{d}} \frac{g_{1}(A)}{|A|}>c n^{2} r_{n}^{2 d+1} .
$$

Proof Since $|A| \leq \beta K_{n}^{d}$, then by Lemma 6.3, we have

$$
|\partial A| \geq(2 d)^{-1}\left(1-\beta^{1 / d}\right)|A|^{(d-1) / d} \geq \frac{\left(1-\beta^{1 / d}\right)|A|}{2 d \beta^{1 / d} K_{n}} .
$$

Combining (45) with Lemma 6.5, w.h.p.

$$
\begin{aligned}
\inf _{|A| \leq \beta K_{n}^{d}} \frac{g_{1}(A)}{|A|} & >\frac{n^{2}}{K_{n}^{2 d}}\left(H_{-}^{-1}\left(\frac{1}{2}+\frac{(d+3)^{d / 2}}{2 \alpha \varepsilon^{d}}\right)\right)^{2} \inf _{|A| \leq \beta K_{n}^{2}} \frac{|\partial A|}{|A|} \\
& \geq \frac{n^{2}\left(1-\beta^{1 / d}\right)}{\beta^{1 / d} K_{n}^{2 d+1}}\left(H_{-}^{-1}\left(\frac{1}{2}+\frac{(d+3)^{d / 2}}{2 \alpha \varepsilon^{d}}\right)\right)^{2}
\end{aligned}
$$

which implies our result.

For $F \subseteq\{1,2, \cdots, n\}$ and $F^{c}=\{1,2, \cdots, n\} \backslash F$, set

$$
\Phi_{n}:=\inf _{|F| \leq \frac{n}{2}, t>0}\left\{\frac{1}{|F|} \sum_{i \in F, j \in F^{c}} f_{n}\left(\left\|X_{i}(t)-X_{j}(t)\right\|\right)\right\} .
$$

We have the following lemma.

Lemma 6.7 Assume that $\alpha \in\left(\frac{2^{d-1}}{d \pi_{d}}, \infty\right]$, and that there exists a constant $\delta>0$ such that (25) holds. For any constant $\varepsilon>0$, there exists a constant $c=c(\alpha, \varepsilon, d)>0$ such that 
(i) if $\alpha \varepsilon^{d} \leq(d+3)^{d / 2}$, then w.h.p.

$$
\Phi_{n} \geq c \min \left\{n r_{n}^{d+1} f_{n}\left((\delta+\varepsilon) r_{n}\right), \frac{f_{n}\left(R_{c}+(\delta+\varepsilon) r_{n}\right)}{(\log n)^{(2 d-1) /(d-1)}}\right\} ;
$$

(ii) if $\alpha \varepsilon^{d}>(d+3)^{d / 2}$, then w.h.p.

$$
\Phi_{n} \geq c n f_{n}\left((\delta+\varepsilon) r_{n}\right) \min \left\{r_{n}^{d+1}, 1\right\} .
$$

Proof (i) For $F \subseteq\{1,2, \cdots, n\}$, define $\widetilde{F}:=\left\{X_{i}(0): i \in F\right\} \subseteq \mathcal{X}_{n}$ to be the initial positions of agents whose indexes are in $F$. For $D_{1}, D_{2} \subset[0,1]^{d}$, set

$$
g_{D_{1}, D_{2}}(F)=g_{D_{1}, D_{2}, n}(F):=\sum_{x \in D_{1} \cap \widetilde{F}, y \in D_{2} \cap \widetilde{F^{c}}} f_{n}\left(\|x-y\|+\delta r_{n}\right) .
$$

Take $g(F)=g_{[0,1]^{d},[0,1]^{d}}(F)$, and define

$$
\Phi_{n}^{\prime}:=\inf _{|F| \leq n / 2} \frac{\sum_{i \in F, j \in F^{c}} f_{n}\left(\left\|X_{i}(0)-X_{j}(0)\right\|+\delta r_{n}\right)}{|F|}=\inf _{|F| \leq n / 2} \frac{g(F)}{|F|} .
$$

Now, we estimate $\Phi_{n}^{\prime}$. Denote

$$
A_{F}:=\left\{z_{i}:\left|S_{i} \cap \widetilde{F}\right|>\frac{1}{2}\left|S_{i} \cap \mathcal{X}_{n}\right|\right\} \subseteq B_{\mathbb{Z}}\left(K_{n}\right)
$$

and

$$
\widetilde{A_{F}}:=\bigcup_{z_{i} \in A_{F}} S_{i} \cap \mathcal{X}_{n}
$$

Set $\beta:=1-\frac{1}{4 c_{1}(d+4)^{d / 2}}$. If $\left|A_{F}\right|>\beta K_{n}^{d}$, then $\left|A_{F}^{c}\right| \leq(1-\beta) K_{n}^{d}$. By Lemma 6.2, we have w.h.p.

$$
\sum_{z_{i} \in A_{F}^{c}}\left|S_{i} \cap \mathcal{X}_{n}\right| \leq c_{1} \varepsilon^{d}\left|A_{F}^{c}\right| n r_{n}^{d}<\frac{n}{4}
$$

where $c_{1}$ is the constant $c$ appearing in Lemma 6.2. If $|F| \leq n / 2$, then $\left|\widetilde{F^{c}}\right|=\left|F^{c}\right|>\frac{n}{2}$. Thus, there exist at least $\frac{n}{4}$ vertices in $\widetilde{F^{c}}$ contained by $\widetilde{A_{F}}$. For $x \in \widetilde{F^{c}} \cap \widetilde{A_{F}}$, without loss of generality we assume that $x \in S_{i}$ with $z_{i} \in A_{F}$. Then by the definition of $A_{F}$ we can get $\left|\widetilde{F} \cap S_{i}\right| \geq\left|\widetilde{F^{c}} \cap S_{i}\right| \geq 1$, which indicates that there exists at least one vertex $y$ such that $y \in \widetilde{F} \cap S_{i}$. Note that the length of the side of $S_{i}$ is less than $\varepsilon r_{n} / \sqrt{d+3}$. Then w.h.p.

$$
\inf _{|F| \leq \frac{n}{2},\left|A_{F}\right|>\beta K_{n}^{d}} \frac{g(F)}{|F|} \geq \frac{\frac{n}{4} f_{n}\left(\frac{\varepsilon r_{n} \sqrt{d}}{\sqrt{d+3}}+\delta r_{n}\right)}{n / 2} \geq \frac{f_{n}\left((\varepsilon+\delta) r_{n}\right)}{2} .
$$

In the following we consider the case of $\left|A_{F}\right| \leq \beta K_{n}^{d}$. Let $A_{1}, A_{2}, \cdots, A_{m_{F}}$ be components of $A_{F}$ satisfying: 1) $A_{1}, A_{2}, \cdots, A_{m_{F}}$ are all $\left\lceil\frac{\sqrt{d+3}}{\varepsilon}\right\rceil$-connected; 2) $A_{i} \cup A_{j}, 1 \leq i \neq j \leq m_{F}$ is not $\left\lceil\frac{\sqrt{d+3}}{\varepsilon}\right\rceil$-connected; 3) $\left|A_{1}\right| \geq\left|A_{2}\right| \geq \cdots \geq A_{m_{F}}$. Without loss of generality, we assume that 
$\left|A_{i}\right| \geq \gamma(\log n)^{d /(d-1)}$ for $1 \leq i \leq i_{F}$, and $\left|A_{i}\right|<\gamma(\log n)^{d /(d-1)}$ for $i_{F}+1 \leq i \leq m_{F}$, where $i_{F} \in\left[1, m_{F}\right]$ and $\gamma$ is the same constant appearing in Lemma 6.4. By Lemma 6.4, we have

$$
\inf _{\left|A_{F}\right| \leq \beta K_{n}^{d}, 1 \leq i \leq i_{F}} \frac{g_{1}\left(A_{i}\right)}{\left|A_{i}\right|} \geq \frac{c_{2} n^{2} r_{n}^{2 d}}{K_{n}} \quad \text { w.h.p. }
$$

where $c_{2}$ is the constant $c$ appearing in Lemma 6.4.

For $i \in\left[1, i_{F}\right]$, it is easy to see that if $z_{k} \in A_{i}$ and $z_{j} \in A_{i}^{c}$ with $z_{k} \sim z_{j}$, then $z_{j} \in A_{F}^{c}$, and the distance of any pair of vertices in $S_{k} \cup S_{j}$ is not greater than $\varepsilon r_{n}$. By the definition of $A_{F}$, we have

$$
g_{S_{k}, S_{j}}(F)=\sum_{x \in S_{k} \cap \widetilde{F}, y \in S_{j} \cap \widetilde{F^{c}}} f_{n}(\|x-y\|) \geq \frac{f_{n}\left((\varepsilon+\delta) r_{n}\right)}{4}\left|\mathcal{X}_{n} \cap S_{k}\right| \cdot\left|\mathcal{X}_{n} \cap S_{j}\right| .
$$

Therefore, if $\mathcal{P}_{\theta(n)} \subseteq \mathcal{X}_{n}$, then

$$
\sum_{z_{k} \in A_{i}, z_{j} \in A_{F}^{c}, z_{k} \sim z_{j}} g_{S_{k}, S_{j}}(F)=\sum_{z_{k} \in A_{i}, z_{j} \in A_{i}^{c}, z_{k} \sim z_{j}} g_{S_{k}, S_{j}}(F) \geq \frac{f_{n}\left((\varepsilon+\delta) r_{n}\right)}{4} g_{1}\left(A_{i}\right) .
$$

Moreover, by (44), we see that $\mathcal{P}_{\theta(n)} \subseteq \mathcal{X}_{n}$ holds w.h.p.

Set

$$
S_{F}^{1}:=\bigcup_{i=1}^{i_{F}} \bigcup_{z_{k} \in A_{i}} S_{k}
$$

By (48), we have w.h.p.

$$
g_{S_{F}^{1},[0,1]^{d} \backslash S_{F}^{1}}(F) \geq \sum_{i=1}^{i_{F}} \sum_{z_{k} \in A_{i}, z_{j} \in A_{F}^{c}, z_{k} \sim z_{j}} g_{S_{k}, S_{j}}(F) \geq \sum_{i=1}^{i_{F}} \frac{f_{n}\left((\varepsilon+\delta) r_{n}\right)}{4} g_{1}\left(A_{i}\right) .
$$

For $i \in\left[i_{F}+1, m_{F}\right]$, if $\bigcup_{z_{j} \in A_{i}} S_{j} \cap \widetilde{F^{c}} \neq \emptyset$, then we have $g_{D_{i}^{\prime}, D_{i}^{\prime}}(F) \geq f_{n}\left(\varepsilon r_{n}\right)$ where $D_{i}^{\prime}=\bigcup_{z_{j} \in A_{i}} S_{j} ;$ If $\bigcup_{z_{j} \in A_{i}} S_{j} \cap \widetilde{F^{c}}=\emptyset$, then by Lemma 2.1, we know that w.h.p. $G\left(\mathcal{X}_{n} ; R_{c}+\varepsilon r_{n}\right)$ is connected. Thus, there exists at least one vertex $x^{*} \in\left(\bigcup_{z_{j} \in A_{i}} S_{j}\right)^{c} \cap \mathcal{X}_{n}$ such that the set

$$
\left\{y: y \in \bigcup_{z_{j} \in A_{i}} S_{j} \cap \widetilde{F},\left\|x^{*}-y\right\| \leq R_{c}+\varepsilon r_{n}\right\}
$$

is not empty. Assume that $x^{*} \in S_{k}\left(1 \leq k \leq K_{n}^{d}\right)$ and $z_{k}$ is the corresponding integer point of $S_{k}$, then $z_{k}$ must be $\left\lceil\frac{\sqrt{d+3}}{\varepsilon}\right\rceil$-connected with $A_{i}$, and $z_{k} \in A_{F}^{c}$. Denote $D_{i}^{\prime \prime}=\bigcup_{z_{j} \in A_{i}} S_{j} \cup S_{k}$. If $x^{*} \in \widetilde{F^{c}}$, then

$$
g_{D_{i}^{\prime \prime}, D_{i}^{\prime \prime}}(F) \geq f_{n}\left(R_{c}+(\delta+\varepsilon) r_{n}\right) ;
$$

Otherwise, by the definition of $A_{F}$ we have $S_{k} \cap \widetilde{F^{c}} \neq \emptyset$, and

$$
g_{D_{i}^{\prime \prime}, D_{i}^{\prime \prime}}(F) \geq g_{S_{k}, S_{k}}(F) \geq f_{n}\left((\varepsilon+\delta) r_{n}\right) .
$$


Set

$$
S_{F}^{2}:= \begin{cases}\bigcup_{i=i_{F}+1}^{m_{F}} D_{i}^{\prime}, & \text { if } \bigcup_{z_{j} \in A_{i}} S_{j} \cap \widetilde{F^{c}} \neq \emptyset, \\ \bigcup_{i=i_{F}+1}^{m_{F}} D_{i}^{\prime \prime}, & \text { otherwise. }\end{cases}
$$

For $z \in \mathbb{Z}^{d}$, it is easy to see that the number of different $\left\lceil\frac{\sqrt{d+3}}{\varepsilon}\right\rceil$-connected components which is $\left\lceil\frac{\sqrt{d+3}}{\varepsilon}\right\rceil$-connected with $z$ is less than $\left\lceil\frac{\sqrt{d+3}}{\varepsilon}\right\rceil^{d}$. By the above argument we have w.h.p.

$$
g_{S_{F}^{2}, S_{F}^{2}}(F) \geq\left\lceil\frac{\sqrt{d+3}}{\varepsilon}\right\rceil^{-d}\left(m_{F}-i_{F}\right) f_{n}\left(R_{c}+(\delta+\varepsilon) r_{n}\right) .
$$

Let $S_{F}^{3}=[0,1]^{d} \backslash\left(S_{F}^{1} \cup S_{F}^{2}\right)$. For $x \in S_{F}^{3} \cap \widetilde{F}$, we assume that $x \in S_{k}\left(1 \leq k \leq K_{n}^{d}\right)$, and denote $z_{k} \in B_{\mathbb{Z}}\left(K_{n}\right)$ as the corresponding integer point of $S_{k}$. It is clear that $z_{k} \in A_{F}^{c}$, and the set $S_{k} \cap \widetilde{F^{c}}$ is not empty. Thus,

$$
g_{S_{F}^{3}, S_{F}^{3}}(F) \geq \sum_{x \in S_{F}^{3} \cap \widetilde{F}} f_{n}\left((\delta+\varepsilon) r_{n}\right)=\left|S_{F}^{3} \cap \widetilde{F}\right| f_{n}\left((\delta+\varepsilon) r_{n}\right) .
$$

By the definition of $S_{F}^{1}$ and $S_{F}^{2}$ we have $\operatorname{Leb}\left(S_{F}^{1} \cap S_{F}^{2}\right)=0$. By (49), (50) and (51), we have w.h.p.

$$
\begin{aligned}
g(F) \geq & g_{S_{F}^{1},[0,1]^{d} \backslash S_{F}^{1}}(F)+g_{S_{F}^{2}, S_{F}^{2}}(F)+g_{S_{F}^{3}, S_{F}^{3}}(F) \\
\geq & \sum_{i=1}^{i_{F}} \frac{f_{n}\left((\varepsilon+\delta) r_{n}\right)}{4} g_{1}\left(A_{i}\right)+\left|S_{F}^{3} \cap \widetilde{F}\right| f_{n}\left((\delta+\varepsilon) r_{n}\right) \\
& +\left\lceil\frac{\sqrt{d+3}}{\varepsilon}\right]^{-d}\left(m_{F}-i_{F}\right) f_{n}\left(R_{c}+(\delta+\varepsilon) r_{n}\right) .
\end{aligned}
$$

By the above inequality, we have w.h.p.

$$
\begin{aligned}
\inf _{\left|A_{F}\right| \leq \beta K_{n}^{d}} \frac{g(F)}{|F|} & =\inf _{\left|A_{F}\right| \leq \beta K_{n}^{d}} \frac{g(F)}{\left|S_{F}^{1} \cap \widetilde{F}\right|+\left|S_{F}^{2} \cap \widetilde{F}\right|+\left|S_{F}^{3} \cap \widetilde{F}\right|} \\
& \geq \inf _{\left|A_{F}\right| \leq \beta K_{n}^{d}} \frac{g(F)}{c_{1} \varepsilon^{d} n r_{n}^{d}\left(\sum_{i=1}^{i_{F}}\left|A_{i}\right|+\left(m_{F}-i_{F}\right) \gamma(\log n)^{d /(d-1)}\right)+\left|S_{F}^{3} \cap \widetilde{F}\right|} \\
& \geq \min \left\{\frac{\frac{f_{n}\left((\varepsilon+\delta) r_{n}\right)}{4} \sum_{i=1}^{i_{F}} g_{1}\left(A_{i}\right)}{c_{1} \varepsilon^{d} n r_{n}^{d} \sum_{i=1}^{i_{F}}\left|A_{i}\right|}, \frac{\left\lceil\frac{\sqrt{d+3}}{\varepsilon}\right\rceil^{-d} f_{n}\left(R_{c}+(\delta+\varepsilon) r_{n}\right)}{c_{1} \varepsilon^{d} n r_{n}^{d} \gamma(\log n)^{d /(d-1)}}, f_{n}\left((\delta+\varepsilon) r_{n}\right)\right\} \\
& \geq \min \left\{\frac{f_{n}\left((\varepsilon+\delta) r_{n}\right) c_{2} n^{2} r_{n}^{2 d}}{4 c_{1} \varepsilon^{d} n r_{n}^{d} K_{n}}, \frac{f_{n}\left(R_{c}+(\delta+\varepsilon) r_{n}\right)}{\left\lceil\frac{\sqrt{d+3}}{\varepsilon}\right\rceil^{d} c_{1} \varepsilon^{d} n r_{n}^{d} \gamma(\log n)^{d /(d-1)}}\right\},
\end{aligned}
$$

where (47) is used in the last inequality. Combining this with (46), we obtain that there exists a constant $c>0$ such that

$$
\Phi_{n}^{\prime} \geq c \min \left\{n r_{n}^{d+1} f_{n}\left((\delta+\varepsilon) r_{n}\right), \frac{f_{n}\left(R_{c}+(\delta+\varepsilon) r_{n}\right)}{(\log n)^{(2 d-1) /(d-1)}}\right\} \quad \text { w.h.p. }
$$


By (25), we have for $t>0$,

$$
\left\|X_{i}(t)-X_{j}(t)\right\| \leq\left\|X_{i}(0)-X_{j}(0)\right\|+\delta r_{n}, \quad \text { w.h.p. }
$$

Hence, $\Phi_{n} \geq \Phi_{n}^{\prime}$ holds w.h.p. Combining this with (52) yields our result.

(ii) The definition of $c_{1}, \beta$ and $A_{F}$ is the same as that in (i). By (46) and Lemma 6.5, we have

$$
\begin{aligned}
\operatorname{linf}_{|F| \leq \frac{n}{2},\left|A_{F}\right|>\beta K_{n}^{d}} \frac{g(F)}{|F|} & \geq \frac{\frac{n}{4} \cdot \frac{n}{2 K_{n}^{d}} H_{-}^{-1}\left(\frac{1}{2}+\frac{(d+3)^{d / 2}}{2 \alpha \varepsilon^{d}}\right) f_{n}\left(\frac{\varepsilon r_{n} \sqrt{d}}{\sqrt{d+3}}+\delta r_{n}\right)}{n / 2} \\
& \geq \frac{n H_{-}^{-1}\left(\frac{1}{2}+\frac{(d+3)^{d / 2}}{2 \alpha \varepsilon^{d}}\right) f_{n}\left((\varepsilon+\delta) r_{n}\right)}{4 K_{n}^{d}} \quad \text { w.h.p. }
\end{aligned}
$$

For the case of $\left|A_{F}\right| \leq \beta K_{n}^{d}$, by Lemmas 6.2 and 6.6 we have

$$
\inf _{|F| \leq \frac{n}{2},\left|A_{F}\right| \leq \beta K_{n}^{d}} \frac{g(F)}{|F|} \geq \frac{f_{n}\left((\varepsilon+\delta) r_{n}\right)}{c_{1} \varepsilon^{d} n r_{n}^{d}} \inf _{\left|A_{F}\right| \leq \beta K_{n}^{d}} \frac{g_{1}(F)}{\left|A_{F}\right|} \geq \frac{c_{3} n^{2} r_{n}^{2 d+1}}{c_{1} \varepsilon^{d} n r_{n}^{d}} f_{n}\left((\varepsilon+\delta) r_{n}\right) \quad \text { w.h.p. }
$$

where $c_{3}$ is the same constant as $c$ appearing in Lemma 6.6. By (53) and the fact $\Phi_{n} \geq \Phi_{n}^{\prime}$ yields our result.

Proof of Lemma 5.3 Suppose $\bar{k}_{n, \delta} \leq 1-\varepsilon$. By (25), we have for $t>0$,

$$
\left\|X_{i}(t)-X_{j}(t)\right\| \geq\left\|X_{i}(0)-X_{j}(0)\right\|-\delta r_{n}, \quad \text { w.h.p. }
$$

By $(23) \Delta_{n}(t) \leq \Delta_{n, \delta}$ holds w.h.p. for all $t>0$. By Lemma 5.2, we see that w.h.p. $\Delta_{n}(t) \leq$ $1-\frac{2 \varepsilon}{3}$ holds for all $t>0$. Thus, given $\lambda \in \mathbb{R}$, if $\lambda<\frac{\varepsilon}{2}-1$, then w.h.p. $P(t)-\lambda I_{n}$ is a strictly diagonally dominant matrix and $\operatorname{det}\left(P(t)-\theta I_{n}\right) \neq 0$ for all $t>0$, which indicates that $\lambda$ is not an eigenvalue of $P(t)$. Thus, w.h.p.

$$
\lambda_{n}(t) \geq \frac{\varepsilon}{2}-1, \quad \forall t>0 .
$$

On the other hand, note that $P(t)$ is a symmetric stochastic matrix, then the stationary distribution of $P(t)$ is $\left(\frac{1}{n}, \frac{1}{n}, \cdots, \frac{1}{n}\right)$. Therefore for $t>0$, the Cheeger' constant of $P(t)$ is not less than $\Phi_{n}$. By the Cheeger's inequality (Proposition 6 in [33]), we have $\lambda_{2}(t) \leq 1-\Phi_{n}^{2}$ for $t>0$. Combining this with Lemma 6.7 and (54), our results can be deduced. 